\title{
SPATIAL CLUSTERING FROM GALEX-SDSS SAMPLES: STAR FORMATION HISTORY AND LARGE-SCALE CLUSTERING
}

\author{
Sébastien Heinis ${ }^{1}$, Tamás Budavári ${ }^{1}$, Alex S. Szalay ${ }^{1}$, Stéphane Arnouts ${ }^{2}$, Miguel A. Aragón-Calvo ${ }^{1}$, Ted \\ K. Wyder ${ }^{3}$, Tom A. Barlow ${ }^{3}$, Karl Foster ${ }^{3}$, Friedman G. Peter ${ }^{3}$, D. Christopher Martin ${ }^{3}$, Patrick Morrissey ${ }^{3}$, Susan \\ G. NefF $^{4}$, David Schiminovich ${ }^{5}$, Mark Seibert ${ }^{6}$, Luciana Bianchi ${ }^{1}$, José Donas ${ }^{7}$, Timothy M. Heckman ${ }^{1}$, \\ Young-Wook Lee ${ }^{8}$, Barry F. Madore ${ }^{6}$, Bruno Milliard ${ }^{7}$, R. Michael Rich ${ }^{9}$, and Sukyoung K. Yi ${ }^{8}$ \\ ${ }^{1}$ Department of Physics and Astronomy, The Johns Hopkins University, Homewood Campus, Baltimore, MD 21218, USA \\ ${ }^{2}$ Canada-France-Hawaii Telescope Corporation, Kamuela, HI 96743, USA \\ ${ }^{3}$ California Institute of Technology, MC 405-47, 1200 East California Boulevard, Pasadena, CA 91125, USA \\ ${ }^{4}$ Laboratory for Astronomy and Solar Physics, NASA Goddard Space Flight Center, Greenbelt, MD 20771, USA \\ ${ }^{5}$ Department of Astronomy, Columbia University, New York, NY 10027, USA \\ ${ }^{6}$ Observatories of the Carnegie Institution of Washington, 813 Santa Barbara Street, Pasadena, CA 91101, USA \\ ${ }^{7}$ Laboratoire d'Astrophysique de Marseille-CNRS, Pôle de l'Étoile Site de Château-Gombert, 38, rue Frédéric Joliot-Curie 13388 Marseille cedex 13, France \\ ${ }^{8}$ Center for Space Astrophysics, Yonsei University, Seoul 120-749, Korea \\ ${ }^{9}$ Department of Physics and Astronomy, University of California, Los Angeles, CA 90095, USA \\ Received 2008 December 8; accepted 2009 April 21; published 2009 June 4
}

\begin{abstract}
We measure the projected spatial correlation function $w_{p}\left(r_{p}\right)$ from a large sample combining Galaxy Evolution Explorer ultraviolet imaging with the Sloan Digital Sky Survey spectroscopic sample. We study the dependence of the clustering strength for samples selected on $(N U V-r)_{\text {abs }}$ color, specific star formation rate (SSFR), and stellar mass. We find that there is a smooth transition in the clustering of galaxies as a function of this color from weak clustering among blue galaxies to stronger clustering for red galaxies. The clustering of galaxies within the "green valley" has an intermediate strength, and is consistent with that expected from galaxy groups. The results are robust to the correction for dust extinction. The comparison with simple analytical modeling suggests that the halo occupation number increases with older star formation epochs. When splitting according to SSFR, we find that the SSFR is a more sensitive tracer of environment than stellar mass.
\end{abstract}

Key words: ultraviolet: galaxies

Online-only material: color figures

\section{INTRODUCTION}

The nature of star-forming galaxies has deeply changed with the evolution of the universe. Besides the strong decrease in the cosmic star formation rate (SFR) since $z \sim 1$ (e.g., Hopkins \& Beacom 2006; Schiminovich et al. 2005), the bulk of star formation has shifted from high stellar mass to low stellar mass systems, ("downsizing," see Bundy et al. 2006; Cowie et al. 1996; Juneau et al. 2005), a phenomenon which exhibits a similar trend in dark matter halo mass (Heinis et al. 2007). In the local universe results from optical surveys show that the galaxy population is bimodal, divided at a stellar mass of $\sim 3 \times 10^{10} M_{\odot}$ between actively star-forming bluesequence galaxies and passively evolving red-sequence galaxies (Kauffmann et al. 2003a). This bimodality in galaxy properties has been observed from low redshift up to $z \sim 1.5$ (Bell et al. 2004; Cirasuolo et al. 2007; Cassata et al. 2008; Franzetti et al. 2007) although the proportion between the red and blue sequence has been changing. Several studies indeed show that the density of red galaxies has been increasing since $z=2$ while the density of galaxies on the blue sequences remained roughly constant (Bell et al. 2004; Faber et al. 2007). These are further pieces of evidence that some previously star-forming galaxies have quenched their star formation and moved from the blue to the red sequence at a rate balanced by ongoing star formation among the remaining blue-sequence galaxies (Arnouts et al. 2007; Martin et al. 2007). Various mechanisms can be involved in the quenching of star formation. Galaxy mergers can trigger active galactic nucleus (AGN) activity (e.g., Hopkins et al. 2007), and hence AGN feedback, which is efficient in further preventing gas cooling (Croton et al. 2006). Supernovae explosions inject energy into the interstellar medium and may expel gas from galaxies in low-mass halos (e.g., Dekel \& Silk 1986). Galaxy environment is also an important factor in this evolution: in clusters for instance, several processes such as starvation (Larson et al. 1980), harassment (Moore et al. 1999) or ram pressure stripping (Gunn \& Gott 1972), which can be effective at large distances from the cluster center (Chung et al. 2007; Tonnesen et al. 2007), may combine to lead to morphological transformations and removal of the gas reservoir. Numerical studies also suggest that accounting for gravitational heating through satellite accretion can reproduce the observed trends between star formation and environment (Khochfar \& Ostriker 2008).

A common tool used to place constraints on the physical mechanisms involved in this evolution has been the study of galaxies' properties and their link with environment within the color-magnitude diagram. Recent studies have showed that at fixed luminosity the fraction of red galaxies increases in denser environments, while the mean color of the red and blue sequences are fairly insensitive to local density (Balogh et al. 2004). Hogg et al. (2003) found that for blue galaxies, at fixed star formation history, stellar mass is weakly correlated with environment, and that luminous and faint red galaxies reside in denser environments. Cucciati et al. (2006) showed that the relation between color and local density evolved between $z=1.5$ and $z=0$, which suggests that the links between galaxy properties and environment observed at the present epoch are not only 
determined by galaxy formation conditions ("nature") but also by contributions from galaxy evolution processes ("nurture").

Extending these studies using Galaxy Evolution Explorer (GALEX; Martin et al. 2005) data, Wyder et al. (2007) showed that the galaxy distribution is less bimodal in ultraviolet (UV) optical color-magnitude diagrams, owing to the greater sensitivity of the UV to star formation and dust. Previous work also showed that there is a very tight correlation between the $(N U V-r)_{\mathrm{abs}}{ }^{10}$ color and star formation history (Salim et al. 2005). UV-optical colors furthermore highlight a galaxy population within the intermediate region between the red and the blue sequences, the so-called "green valley" (Martin et al. 2007).

Schawinski et al. (2007) studied early-type galaxies within the UV-optical color-magnitude diagram; they showed that this population exhibits residual star formation, and that the fraction of UV-bright early-type galaxies is higher in lower density environments. In this paper, we extend this study by using the combination of GALEX imaging with Sloan Digital Sky Survey (SDSS) spectroscopic data to measure the dependence of spatial clustering on the $(N U V-r)_{\text {abs }}$ color, in order to link star formation history with environment, the latter being traced by the large-scale clustering. Here, we use a simple power-law approximation to describe the clustering of the galaxies, in order to estimate the mass of the host dark matter halos.

The outline of this paper is as follows: in Section 2 we present the samples from the GALEX surveys, as well as the internal dust correction procedure. Section 3 deals with the clustering analysis tools, and contains a discussion of the systematic errors previously noticed for clustering measurements from GALEX data (Milliard et al. 2007). We present the results in Section 4, discuss them in Section 5, and conclude in Section 6. Throughout this paper we use the concordance $\Lambda$ CDM cosmology with $\Omega_{m}=0.3$ and $\Omega_{\Lambda}=0.7$; we note $H_{0}=100 h \mathrm{~km} \mathrm{~s}^{-1} \mathrm{Mpc}^{-1}$. We use $h=1$ for clustering computations to facilitate comparisons with other studies, and $h=0.7$ for all absolute magnitudes derivations.

\section{DATA SAMPLES}

We consider observations from two different GALEX surveys: the Medium Imaging Survey (MIS) to use the UV photometry for the overall galaxy population detected by the SDSS spectroscopic survey, and the All sky Imaging Survey (AIS), to build a UV-selected sample. Budavári et al. (2009) described in detail how we built the catalogs and footprints; we only summarize here the main characteristics.

We computed the unique area observed in the GALEX MIS and AIS. The fields target pre-defined positions on the sky. These 47,162 points define disjoint cells by including in each cell those regions of sky closer to the center of that cell than to the center of any other cell, similar to the way in which a Voronoi tessellation is defined. The intersection of the circular field of view of a given field and the targeted cell is the primary region for this field. We use here only objects within the primary resolution of AIS or MIS fields. The union of the primary region from all fields defines the primary footprint of a survey. We then use the formalism of Budavári et al. (2008) to compute the footprint of the GALEX-SDSS overlap.

The GALEX 3rd Release (GR3; Morrissey et al. 2007) data set was cross-matched to the SDSS DR6 (Adelman-McCarthy et al. 2008) using a matching radius of $4^{\prime \prime}$. We used the GALEX sources with one and only one SDSS counterpart

$\overline{10}$ We denote absolute magnitudes by the "abs" subscript.

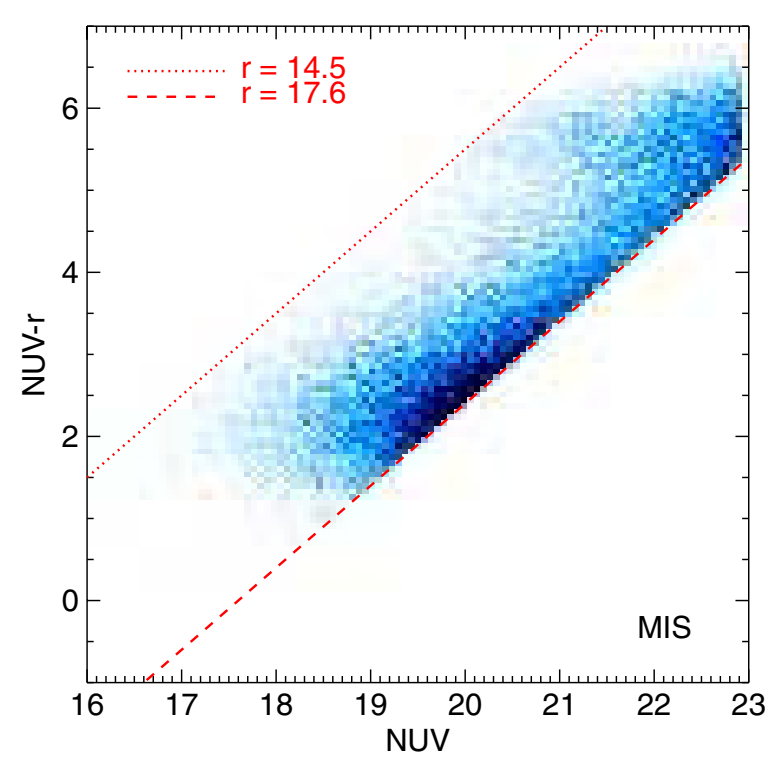

Figure 1. Color-magnitude diagram for MIS selection. The dotted and dashed lines show the cuts in $r$ adopted for SDSS spectroscopic objects.

(A color version of this figure is available in the online journal.)

within the search radius. In the following we only consider SDSS primary sources. We further restricted the SDSS sources to spectroscopically confirmed galaxies with $z<0.3$ and $14.5<r_{\text {petro }}<17.6$. The bright cut avoids the shredded galaxies, while the faint selection ensures a homogeneous spectroscopic completeness over the sky. For GALEX sources, we retained the sources within $0.5 \mathrm{deg}$ of the field centers, as the photometry accuracy decreases beyond this limit, and artifacts concentrate at the edges of the GALEX field of view (Morrissey et al. 2005). We censor sources using GALEX (flagmap values 2 and 4) and SDSS (BLEEDING, BRIGHT_STAR, TRAIL, and HOLE) masks. We use the intersection of the GALEX and SDSS footprints as described in Budavári et al. (2008). We corrected GALEX apparent magnitudes for Galactic extinction using the Schlegel et al. (1998) dust maps and the formulas described in Wyder et al. (2007). We estimated $k$-corrections ${ }^{11}$ at $z=0$ after fitting the seven-band photometry $(F U V, N U V, u, g, r, i$, and $z$ ) to a set of Bruzual \& Charlot (2003) templates ${ }^{12}$; the absolute magnitudes are $k-$ corrected to $z=0$ unless otherwise stated. We also derived $k$-corrections at $z=0.1$ by shifting the filters blueward in wavelength by a factor $1 /(1+z)=1 / 1.1$, in order to compute dust extinction correction (see Section 2.3).

\subsection{MIS/SDSS Selection}

The MIS observations are deep enough to probe the overall population of the SDSS Main Galaxy spectroscopic sample (Strauss et al. 2002). We used MIS fields with exposure times greater than $1000 \mathrm{~s}$ for the $N U V$ band, and objects with $N U V<23$. Figure 1 shows the color-magnitude diagram in the $(N U V, N U V-r)$ plane for the MIS sources. The dashed line corresponds to the faint cut in $r$, and the dotted line to the bright cut we adopt. This sample is obviously not UV-selected, as the density decreases for objects fainter than $r=17.75$, the SDSS target magnitude limit. There is, however, a clear hint of a bimodality in color, with a separation around $N U V-r \sim 4.75$

\footnotetext{
11 Using LePhare: http://www.oamp.fr/people/arnouts/LE_PHARE.html.

12 http://www.oamp.fr/people/arnouts/LE_PHARE/lephare_sed_42GISSEL tar.gz
} 


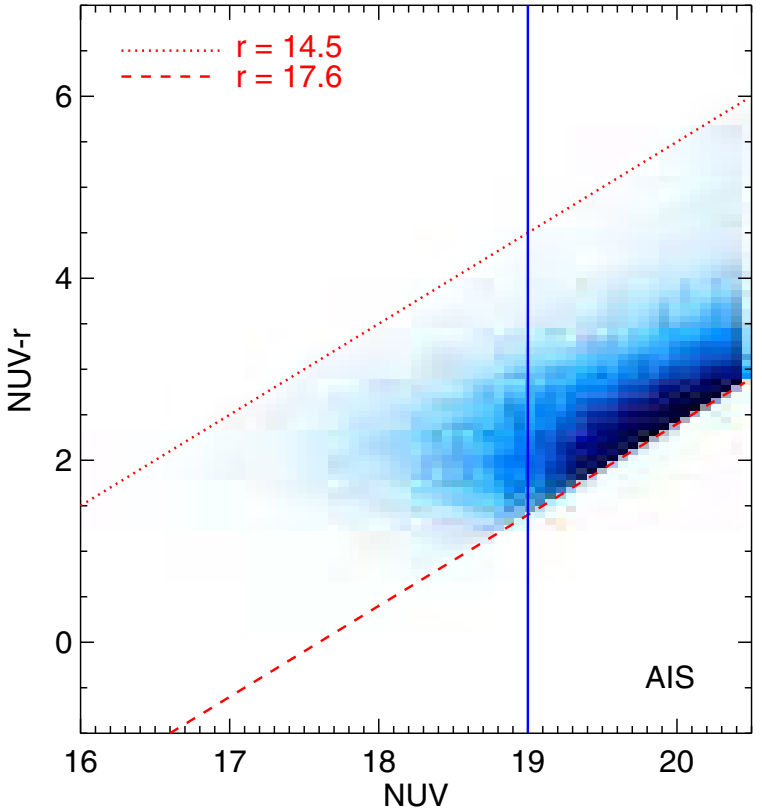

Figure 2. Color-magnitude diagram for AIS selection. The dotted and dashed lines show the cuts in $r$ adopted for SDSS spectroscopic objects, and the solid line the NUV cut chosen to ensure an UV-selected sample.

(A color version of this figure is available in the online journal.)

between the red and the blue sequence. The final sample contains 21,895 galaxies over $598.1 \mathrm{deg}^{2}$.

\subsection{AIS: UV Selection}

We used AIS observations to build UV-selected samples from the GALEX-SDSS spectroscopic samples. Figure 2 shows as before the color-magnitude diagram for AIS objects, with an additional solid line representing the apparent magnitude cut at $N U V=19$ we use to ensure a UV-selected sample. The fraction of objects brighter than $N U V=19$ and fainter than $r=17.6$ that are lost is $3 \%$. We note that the NUV cut removes most of the galaxies on the red sequence; hence our UV-selected galaxy sample mainly contains blue galaxies. The size of the sample is 14,351 galaxies, over an area of $3645.8 \mathrm{deg}^{2}$.

\subsection{Dust Extinction Correction}

In the following, we study the dependence of clustering as a function of the $(N U V-r)_{\text {abs }}$ color, which correlates tightly with star formation history and provides a very good separation between galaxy populations. However, interstellar dust absorbs and scatters stellar light, reddening galaxy colors and interfering with the interpretation of the colors in terms of the star formation history. Several recipes are available to correct for dust reddening, but one often has to rely on various assumptions about the dust content of galaxies. For instance, galaxies with intermediate colors may have redder colors than blue galaxies because of dust, or because of older stellar populations. Hence, applying a given dust extinction correction may imply potential errors for transition or red galaxies. We will therefore present clustering measurements as a function of both non-corrected and corrected colors. We use the relation presented by Johnson et al. (2006):

$$
\begin{aligned}
A_{\mathrm{FUV}} & =1.27-1.56 x+1.35 y-1.24 x y \\
x & =D_{n}(4000)-1.25 \\
y & =(N U V-r)_{\mathrm{abs}, 0.1}-2
\end{aligned}
$$

to estimate the extinction correction in the FUV band, $A_{\mathrm{FUV}}$. Johnson et al. (2006) used the narrowband color $D_{n}(4000)$ from Kauffmann et al. (2003a) to calibrate their relation, while we consider here the $D(4000)$ index directly available from the SDSS DR6 pipeline, which involves the ratio of the flux over a longer wavelength range. We checked that for objects in common between the SDSS DR6 and the sample used by Kauffmann et al. (2003a) the difference between their estimate of $D_{n}(4000)$ and our estimate of $D(4000)$ is reasonably small with a dispersion of $\sim 0.06$. Finally, we did not apply any dust correction to objects without star formation, defined here as those galaxies with $E W(H \alpha)<0$. These objects represent $7 \%$ of the MIS/SDSS sample and less than $1 \%$ of the AIS UVselected one. Negative values of $A_{\text {FUV }}$ according to Equation (1) are set to 0 .

We used the Calzetti et al. (2000) extinction law to convert the extinction in the FUV band into extinction in the NUV, $g$, and $r$ bands with: $A_{\mathrm{NUV}}=0.81 A_{\mathrm{FUV}}, A_{g}=0.46 A_{\mathrm{FUV}}$, and $A_{r}=0.35 A_{\mathrm{FUV}}$.

\section{CLUSTERING ANALYSIS}

We chose to quantify galaxy clustering in our samples using the real-space correlation function. As redshift surveys suffer from redshift distortions due to peculiar velocities, we followed the common practice (Fisher et al. 1994) of measuring first the redshift-space correlation function $\xi\left(r_{p}, \pi\right)$, which is useful for distinguishing between the contribution of galaxy pairs along the line of sight $\pi$ and the direction perpendicular to the line of sight $r_{p}$. We used the Landy \& Szalay (1993) estimator:

$$
\xi\left(r_{p}, \pi\right)=\frac{D D-2 D R+R R}{R R},
$$

where DD, DR, and RR are the data-data, data-random and, random-random pair numbers, respectively. To generate random catalogs, we distributed objects randomly on the sky within the GALEX-SDSS footprint in non-masked areas, which takes care of the angular selection function. We then randomly picked galaxy properties (such as redshift and magnitude) that we assigned to the random objects. This procedure has been shown to provide accurate results, and enabled us to study the dependence of clustering on galaxy properties by fully taking into account selection effects (Li et al. 2006a). While the GALEX-SDSS overlap is fairly patchy, we checked that the integral constraint is negligible for this study.

We used 50 times more random objects than the number of galaxies in the sample under consideration to compute the correlation function. We found out that this is the minimum factor to accurately estimate the correlation function on small scales, given the fairly low statistics of the samples we use here.

As redshift distortions occur only on the line of sight, one can get rid of their effects by computing the projected correlation function

$$
w_{p}\left(r_{p}\right)=2 \int_{0}^{\pi_{\max }} \xi\left(r_{p}, \pi\right) d \pi
$$

After checking for convergence of the integration step, we used here $\pi_{\max }=25 \mathrm{~h}^{-1} \mathrm{Mpc}$. The projected correlation function is finally related to the real-space correlation function $\xi(r)$ by the relation (Davis \& Peebles 1983) 

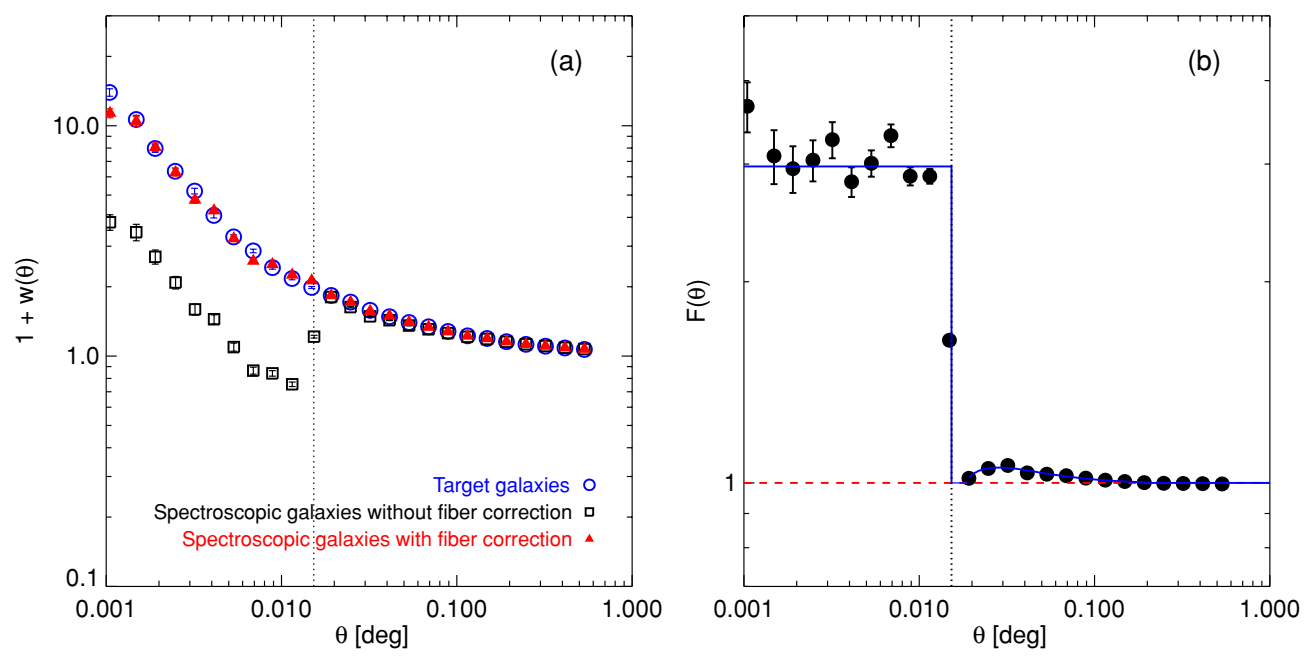

Figure 3. Fiber collision correction. Left: angular correlation function of SDSS target galaxies $\left(w_{t}(\theta)\right.$, open circles), SDSS sources with assigned galaxy spectra $\left(w_{z}(\theta)\right.$, open squares). The filled triangles show the angular correlation function of the SDSS sources with assigned galaxy spectra computed using the correction from fiber collision. Right: the symbols show the ratio $F(\theta)=\left[1+w_{t}(\theta)\right] /\left[1+w_{z}(\theta)\right]$ derived from the measurements shown on the left plot. The solid line shows the functional form we used for this ratio in order to correct for fiber collisions. The dotted line shows the $55^{\prime \prime}$ limit.

(A color version of this figure is available in the online journal.)

$$
w_{p}\left(r_{p}\right)=2 \int_{0}^{\infty} \xi\left(\sqrt{r_{p}^{2}+y^{2}}\right) d y,
$$

where $y$ is the real-space distance along the line of sight.

Under the assumption of a power-law $\xi(r)=\left(r / r_{0}\right)^{-\gamma}$ this relation yields

$$
w_{p}\left(r_{p}\right)=r_{p}\left(\frac{r_{p}}{r_{0}}\right)^{-\gamma} \frac{\Gamma\left(\frac{1}{2}\right) \Gamma\left(\frac{\gamma-1}{2}\right)}{\Gamma\left(\frac{\gamma}{2}\right)} .
$$

We will use the parameters $r_{0}$, the correlation length, and $\gamma$, the slope of the real-space correlation function, to describe the clustering of the galaxies. We use jackknife sampling to derive the error bars on $w_{p}\left(r_{p}\right)$; this procedure has been shown to provide accurate error measurements on $w_{p}\left(r_{p}\right)$ (Zehavi et al. 2005). Specifically, we divided our samples into $N$ separate regions (32 for AIS sample, 20 for MIS), large enough so that their volume is significant. We then computed $w_{p}\left(r_{p}\right)$ for the full sample but removing one jackknife region in turn. For a given bin $r_{p_{i}}$, the errors from these $N$ samples are then given by:

$$
\sigma^{2}\left(w\left(r_{p_{i}}\right)\right)=\frac{N-1}{N} \sum_{j=1}^{N}\left(w^{j}\left(r_{p_{i}}\right)-\left\langle w\left(r_{p_{i}}\right)\right\rangle\right)^{2} .
$$

\subsection{Fiber Collision Correction}

Due to the finite size of the fibers used to take the SDSS spectra, fibers cannot be set closer than $55^{\prime \prime}$, resulting in a lack of close pairs at comoving scales smaller than $\sim 80 h^{-1} \mathrm{kpc}$ (at $z=0.1$ ). We use a statistical approach to correct from this, following Hawkins et al. (2003) and Li et al. (2006a).

We measured the angular correlation function $w_{t}(\theta)$ for the objects classified as galaxies and selected for spectroscopic measurement by the SDSS (i.e., target galaxies, open circles on Figure 3(a)) and for SDSS objects with assigned galaxy spectra $w_{z}(\theta)$ (open squares on Figure 3(a)) within the AIS footprint (large enough to provide an accurate measurement). We then used the ratio

$$
F(\theta)=\frac{1+w_{t}(\theta)}{1+w_{z}(\theta)}
$$

as an estimate for the correction factor needed to correct for fiber collision. The measured values of this ratio are shown in Figure 3(b); we used a functional form for this correction factor represented by the solid lines on Figure 3(b). $F(\theta)$ can be then used to weight the galaxy pairs according to their angular distance. As a test we recomputed the angular correlation function of the SDSS objects with assigned galaxy spectra using this weight (filled triangles on Figure 3(a)); the agreement between this result and the angular correlation function of the target galaxies is excellent.

\subsection{Systematic Errors}

In Milliard et al. (2007) we noticed that the angular correlation function from $G A L E X$ data shows signatures similar to those expected in the case of low-level field-to-field variations. This prevented us from estimating the angular correlation function from the whole data set at once. The approach we used was to estimate the clustering from the angular correlation function by measuring $w(\theta)$ independently for each GALEX field, and then averaging the results. This method cannot be used in the specific case of the GALEX-SDSS spectroscopic samples, given the low statistics. Hence we performed a series of tests to see whether the order of magnitude of the effects on the spatial correlation function is the same as those on the angular correlation function. As in Milliard et al. (2007), we use the Mann-Whitney statistic to determine which GALEX fields should be considered as outliers in terms of UV photometry. We consider potential outliers fields with a $p$-value from the Mann-Whitney test lower than $0.05 .{ }^{13}$ We show in Figure 4 (left) the projected correlation functions $w_{p}\left(r_{p}\right)$ (computed using all data at once) of the full AIS sample ${ }^{14}$ (filled circles), of the fields expected to be outliers ( $p$-value $<0.05$, filled squares, $16 \%$ of the full sample) and the fields that are expected to have an homogeneous UV photometry ( $p$-value $>0.05$, filled triangles $)$. The correlation function of

\footnotetext{
13 This means that the probability that the photometry of these fields comes from the same parent distribution as the other fields is lower than 0.05.

14 The trends are similar for other apparent magnitude cuts and the MIS sample.
} 

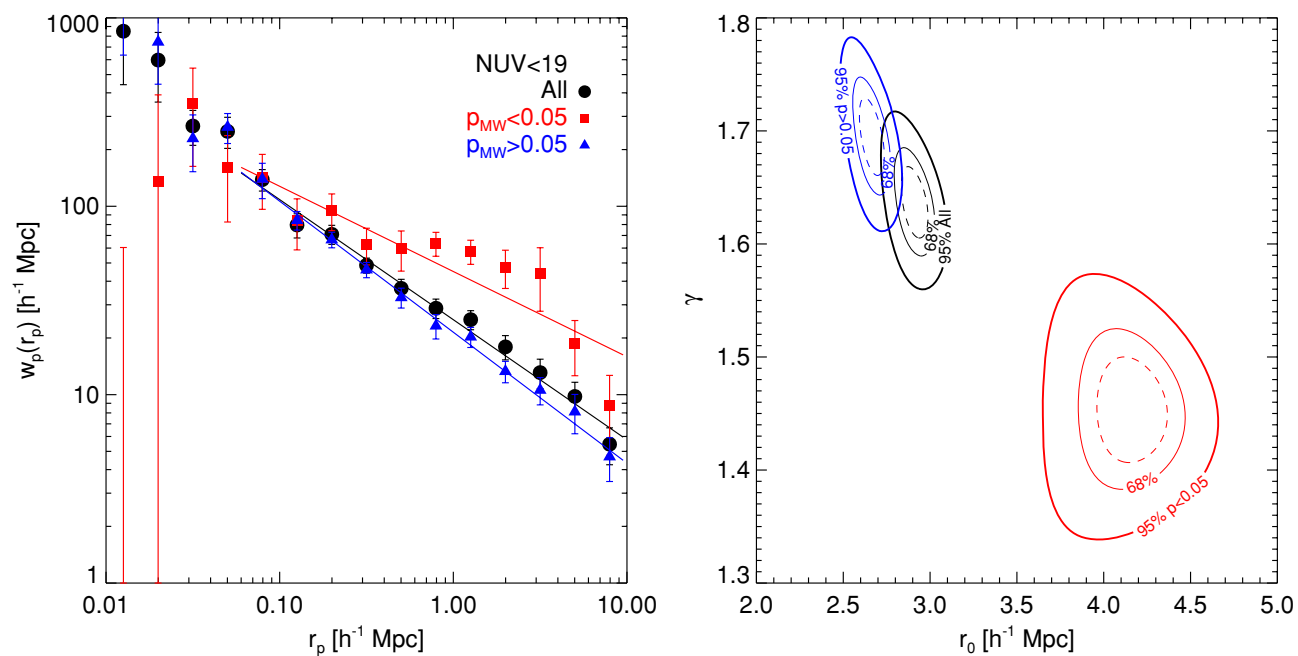

Figure 4. Left: projected correlation function $w_{p}\left(r_{p}\right)$ from the AIS sample with $N U V<19$. Circles show $w_{p}\left(r_{p}\right)$ for the full sample $\left(\right.$ All), squares $w_{p}\left(r_{p}\right)$ for the fields flagged as outliers in terms of UV photometry by the Mann-Whitney statistics $(p<0.05)$, and triangles $w_{p}\left(r_{p}\right)$ for the remaining fields $(p>0.05)$. The solid lines represent the best power law fits. Right: $\chi^{2}$ contours of the power law fits in the $\left(r_{0}, \gamma\right)$ plane; the contours labels include the legend for each correlation function of the left panel plot. Dashed lines show the contour at $\chi_{\min }^{2}+1$, while the thin and thick solid lines show the contours at $68 \%$ and $90 \%$, respectively.

(A color version of this figure is available in the online journal.)

the outlier fields is very different from the fields with a $p$ value $>0.05$, showing a higher amplitude, and a shallower slope. Fitting these results by power laws (see $\chi^{2}$ contours on Figure 4, right) shows that the outlier fields have a correlation length roughly 1.5 times larger than the fields with high $p$-values. These outlier fields have a fairly small impact on the clustering parameters derived for the full sample; excluding or including them from the analysis does not change the conclusions of this study. Nevertheless, these fields indeed show a very different clustering. This stronger clustering may be spurious, or may be real. In the latter case, it might be due to large-scale structure. To further test this hypothesis, we computed $w_{p}\left(r_{p}\right)$ from the entire SDSS data within the AIS footprint, splitting the fields as before, based on the Mann-Whitney results obtained from UV photometry. The trends are similar, with the SDSS data within the footprint of the AIS outlier fields showing a significant stronger clustering. ${ }^{15}$ We also performed the Mann-Whitney test on SDSS data, using the $u$ band; $60 \%$ of the outlier fields are the same as those according to NUV photometry. The clustering derived from the fields classified as outliers with both $u$ and NUV photometry actually dominate the clustering signal observed for the outliers on Figure 4.

As a final test, a friend-of-friend structure finding algorithm applied on SDSS spectroscopic data shows that there is a slight trend for fields with lower $p$-values derived from UV photometry to have larger galaxy groups, as recovered by the friend-of-friend algorithm. These tests indicate that the stronger clustering, as measured by the combination of GALEX and SDSS spectroscopic data, observed for the fields considered as outliers based on the Mann-Whitney test applied on UV photometry is genuine. We checked for instance that the largescale structure known as the "Sloan Great Wall" (Gott et al. 2005 ) is not within our sample; our tests rather show that the fields we classified as outliers contain galaxy groups with larger numbers of members. In consequence, these fields need to be considered in the global analysis. We hereafter use the full

\footnotetext{
15 Trends are also similar for SDSS data within MIS footprint, when splitting fields according to MIS photometry.
}

AIS and MIS samples, and compute the projected correlation function from the whole data set.

\section{RESULTS}

\subsection{Redshift Space Clustering}

Figure 5 shows the redshift space correlation function $\xi\left(r_{p}, \pi\right)$ computed from the MIS sample (left) and the AIS sample (right). The contours show the well known effects due to peculiar velocities (Kaiser 1987): at small scales, galaxies in clusters lead to distortions along the line of sight (fingers of God); this effect is clearly noticed in our MIS/SDSS sample. As expected, this feature is much less prominent in the UV-selected AIS sample, which contains very few red galaxies. At large scales, coherent infall on large structures causes a squashing of the contours, clearly seen in both samples.

\subsection{Clustering as a Function of $(N U V-r)_{\mathrm{abs}}$ Color}

We first consider the MIS sample in order to use the very good separation between red- and blue-sequence galaxies in $(N U V-r)_{\text {abs }}$ color. The distribution of the galaxies in the UV-optical color diagram is shown Figure 6. As noticed by previous studies (Martin et al. 2007; Wyder et al. 2007), the color distribution shows a clear bimodality between the blue and red clouds and a transition population located in the so-called green valley. The distribution of the galaxies in the UV-optical color diagram after applying the dust extinction correction is shown in Figure 7. The shape of the distribution is quite different once this correction is applied, with a less prominent red sequence.

We divided our sample into four bins of $(N U V-r)_{\text {abs }}$ color in the following way. We first set up the green valley for both versions of the color-magnitude diagram $(3.75<$ $(N U V-r)_{\text {abs }}<4.5$ without extinction correction; $2.75<$ $(N U V-r)_{\text {abs }}<3.5$ with extinction correction), following previous studies (see Figures 9 and 25 of Wyder et al. 2007). This also defines a natural cut for the red and the blue populations. We further divided the blue cloud by the median color of this population. The cuts we adopted are shown as dotted lines on Figures 6 and 7. 

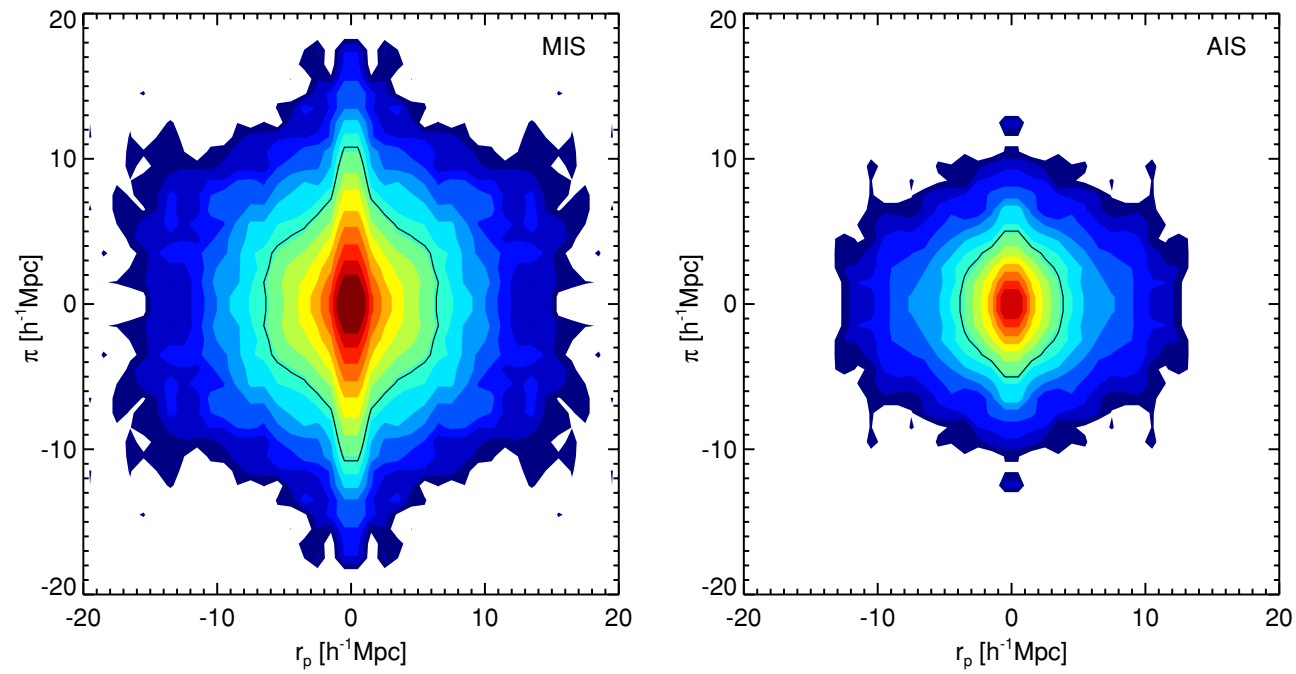

Figure 5. Redshift space correlation functions. Left: contours of $\xi\left(r_{p}, \pi\right)$ for the MIS sample. Right: same as left panel but for the AIS sample. The solid black line shows the contour at $\xi\left(r_{p}, \pi\right)=1$. The contour levels are logarithmically spaced. (A color version of this figure is available in the online journal.)

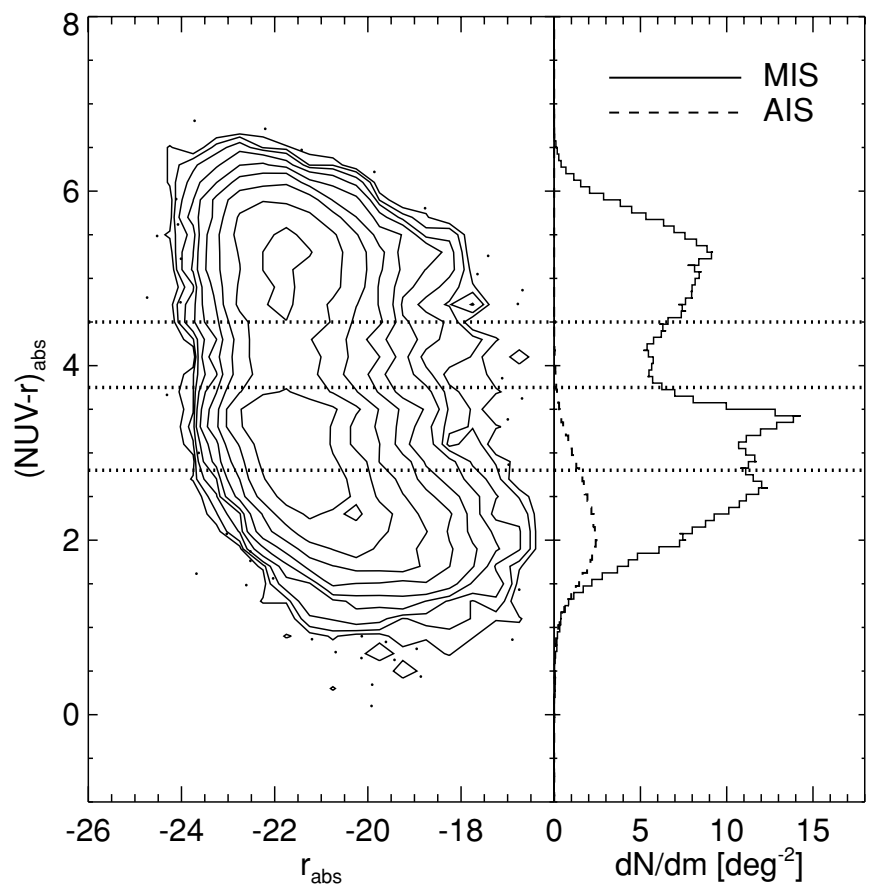

Figure 6. Left: color-magnitude diagram from the MIS/SDSS sample. The dotted lines show the cuts used to measure the projected correlation function. Right: histograms of the $(N U V-r)_{\text {abs }}$ color, with the same cuts shown on the left panel; solid line shows MIS/SDSS sample, and dashed line AIS UV-selected sample.

The correlation functions are shown Figure 8 (for color cuts without dust extinction correction) and Figure 9 (for color cuts with dust extinction correction), as well as the power-law fits. In both cases, there is a monotonic increase of the clustering amplitude for redder galaxies, as well as a steepening of the correlation function. The results obtained without (see clustering parameters in Table 1) or with (Table 2) the dust extinction correction are very similar, while the only criterion to define the color cuts has been to isolate the green valley. This is expected to some extent as the extinction correction we applied is a function of the uncorrected $(N U V-r)_{\text {abs }}$ color and $D(4000)$, which are themselves fairly well correlated with one another (e.g., Martin

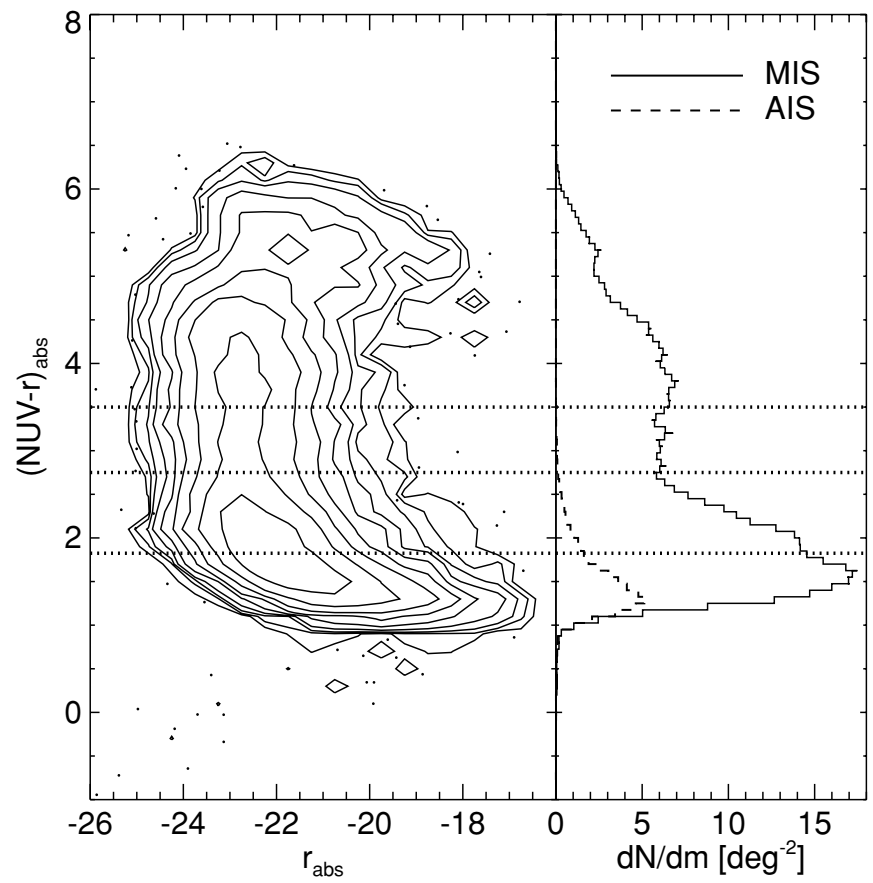

Figure 7. Same as Figure 6 but using dust extinction corrected absolute magnitudes.

et al. 2007; Wyder et al. 2007). Note also that the dust corrected green valley overlaps with the non-dust corrected one at the $50 \%$ level. One could argue that the intermediate clustering of the green valley galaxies we select with non-dust corrected colors is due to a mix of dusty star-forming galaxies and galaxies with older stellar populations. However, the fact that this result holds when using dust corrected colors suggests that dust does not induce such a large population mix in this region of the color-magnitude diagram. Another possibility is that the green valley is actually an intrinsic composite population; Salim et al. (2007) showed that it is built from normal star-forming galaxies, star-forming/AGN composite, and pure AGNs. However, tests show that within the green valley the large-scale clustering of AGNs and non-AGNs is very similar (see Section 5.1). 
Table 1

MIS/SDSS Samples Clustering Results ${ }^{\mathrm{a}}$

\begin{tabular}{lccrrr}
\hline \hline \multicolumn{1}{c}{ Sample } & $N_{\text {gal }}$ & $\langle z\rangle$ & $r_{0}\left(h^{-1} \mathrm{Mpc}\right)$ & \multicolumn{1}{c}{$\gamma$} \\
\hline$-1.0<(N U V-r)_{\mathrm{abs}}<2.8$ & 6293 & 0.08 & $2.93 \pm 0.09$ & $1.58 \pm 0.05$ & $0.66 \pm 0.02$ \\
$2.8<(N U V-r)_{\mathrm{abs}}<3.75$ & 6291 & 0.10 & $4.0 \pm 0.12$ & $1.67 \pm 0.03$ & $0.86 \pm 0.02$ \\
$3.75<(N U V-r)_{\mathrm{abs}}<4.5$ & 2587 & 0.10 & $5.48 \pm 0.21$ & $1.77 \pm 0.04$ & $1.13 \pm 0.04$ \\
$4.5<(N U V-r)_{\mathrm{abs}}<8.0$ & 6702 & 0.10 & $6.93 \pm 0.32$ & $1.84 \pm 0.03$ & $1.43 \pm 0.06$
\end{tabular}

Note. ${ }^{a}$ Without dust extinction correction.

Table 2

MIS/SDSS Samples Clustering Results ${ }^{\mathrm{a}}$

\begin{tabular}{|c|c|c|c|c|c|}
\hline Sample & $N_{\text {gal }}$ & $\langle z\rangle$ & $r_{0}\left(h^{-1} \mathrm{Mpc}\right)$ & $\gamma$ & $b_{8}$ \\
\hline $0 .<(N U V-r)_{\mathrm{abs}, \mathrm{corr}}<1.85$ & 6635 & 0.08 & $3.0 \pm 0.1$ & $1.60 \pm 0.05$ & $0.67 \pm 0.02$ \\
\hline $1.85<(N U V-r)_{\text {abs, corr }}<2.75$ & 6534 & 0.10 & $4.25 \pm 0.12$ & $1.67 \pm 0.03$ & $0.89 \pm 0.02$ \\
\hline $2.75<(N U V-r)_{\mathrm{abs}, \mathrm{corr}}<3.5$ & 2769 & 0.10 & $5.25 \pm 0.24$ & $1.80 \pm 0.05$ & $1.09 \pm 0.05$ \\
\hline $3.5<(N U V-r)_{\mathrm{abs}, \mathrm{corr}}<7.0$ & 5816 & 0.10 & $7.07 \pm 0.32$ & $1.87 \pm 0.03$ & $1.46 \pm 0.06$ \\
\hline
\end{tabular}

Note. ${ }^{\mathrm{a}}$ With dust extinction correction.

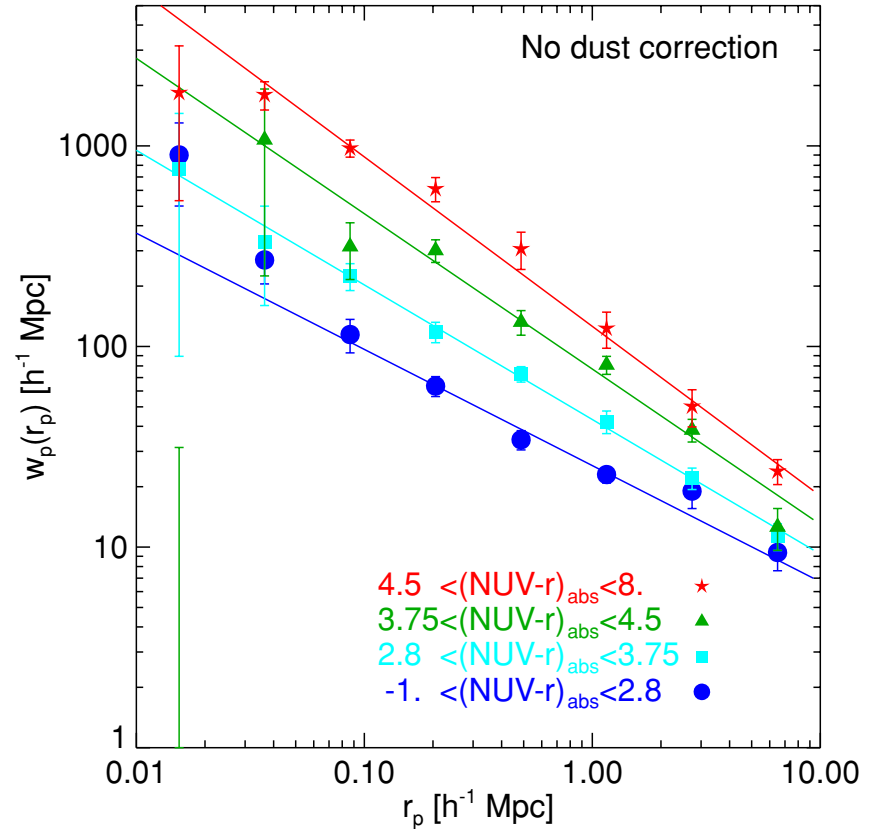

Figure 8. Projected correlation functions of MIS/SDSS samples cut by $(N U V-r)_{\text {abs }}$ color (without dust extinction correction).

(A color version of this figure is available in the online journal.)

We also used the AIS sample to investigate the clustering as a function of the $(N U V-r)_{\text {abs }}$ color within an UV-selected sample. The color distribution for this sample is shown on the right panels of Figures 6 and 7 with dashed lines. We used the same color cuts as for the MIS/SDSS sample and also define three cuts in order to get similar subsample sizes; we use here colors corrected from dust extinction. The correlation functions are presented in Figure 10 and the clustering parameters in Table 3. As observed in our MIS/SDSS sample, the correlation length increases for redder galaxies, although our UV-selected sample is built mainly from galaxies on the blue sequence (see Section 2.2). This increase is not observed if we use the same cuts as for the MIS/SDSS sample. The AIS correlation function for the $1.85<(N U V-r)_{\mathrm{abs}}<2.75$ bin is noisy because of small statistics.

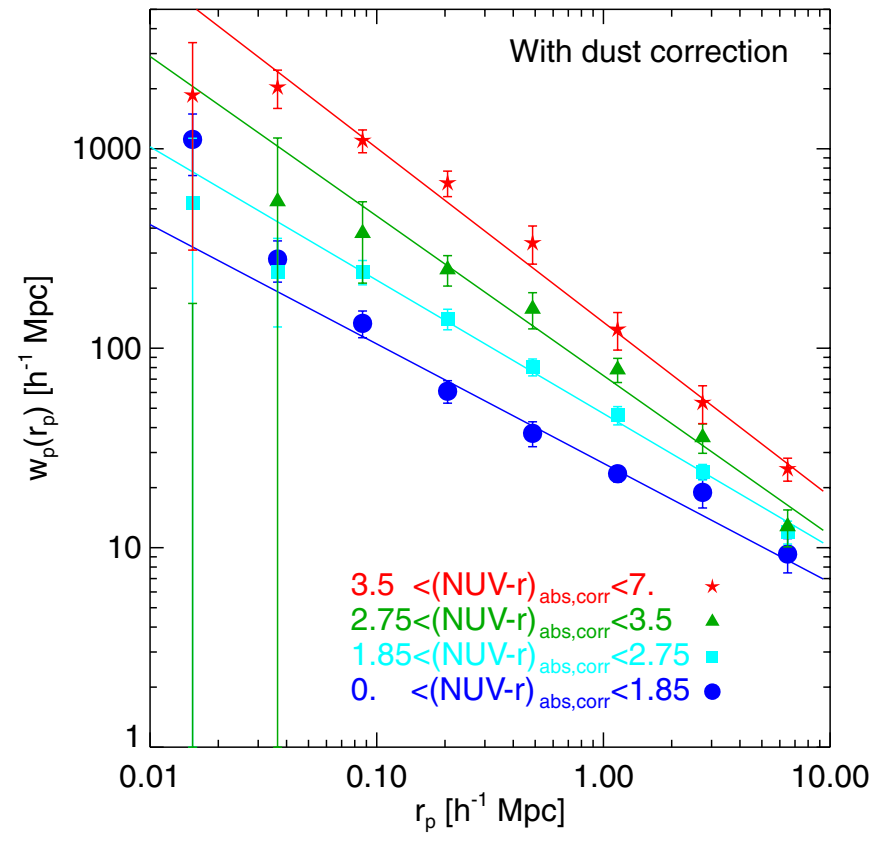

Figure 9. Projected correlation functions of MIS/SDSS sample cut by $(N U V-r)_{\text {abs }}$ color (with dust extinction correction).

(A color version of this figure is available in the online journal.)

Interestingly, our results are poorly fitted by a simple power law. In particular, at scales $r_{p} \lesssim 0.1 h^{-1} \mathrm{Mpc}$, the correlation functions for the blue galaxies in both the MIS and AIS samples show an excess of pairs, while on the contrary the correlation function of our reddest bin shows a lack of satellites (Figures 8 and 9). We note that for the red galaxies, we are biased against low masses and low SSFRs objects (see Figure 15). These faint red galaxies are known to exhibit a strong clustering at small scales (Zehavi et al. 2005). For the blue galaxies, our results suggest a higher satellite fraction for bluer UV galaxies, which is similar to what has been observed at high redshift from UVselected samples (Lee et al. 2006; Ouchi et al. 2005).

In Figure 11, we show the combination of our results for both the AIS (filled and open squares) and MIS samples (filled circles). Thanks to the cut in $(N U V-r)_{\text {abs }}$ color, we are 
Table 3

UV-selected Samples Clustering Results

\begin{tabular}{|c|c|c|c|c|c|}
\hline Sample & $N_{\text {gal }}$ & $\langle z\rangle$ & $r_{0}\left(h^{-1} \mathrm{Mpc}\right)$ & $\gamma$ & $b_{8}$ \\
\hline $0 .<(N U V-r)_{\mathrm{abs}, \mathrm{corr}}<1.85$ & 10861 & 0.05 & $2.59 \pm 0.11$ & $1.69 \pm 0.05$ & $0.57 \pm 0.02$ \\
\hline $1.85<(N U V-r)_{\mathrm{abs}, \mathrm{corr}}<2.75$ & 2596 & 0.06 & $2.34_{-0.40}^{+0.27}$ & $1.51 \pm 0.12$ & $0.56_{-0.07}^{+0.05}$ \\
\hline $0 .<(N U V-r)_{\mathrm{abs}, \mathrm{corr}}<1.35$ & 4507 & 0.05 & $1.79 \pm 0.17$ & $1.55 \pm 0.10$ & $0.45 \pm 0.04$ \\
\hline $1.35<(N U V-r)_{\mathrm{abs}, \mathrm{corr}}<1.64$ & 4503 & 0.05 & $2.07 \pm 0.22$ & $1.51 \pm 0.10$ & $0.51 \pm 0.04$ \\
\hline $1.64<(N U V-r)_{\mathrm{abs}, \mathrm{corr}}<4$ & 4592 & 0.05 & $2.75 \pm 0.16$ & $1.54 \pm 0.06$ & $0.62 \pm 0.03$ \\
\hline
\end{tabular}

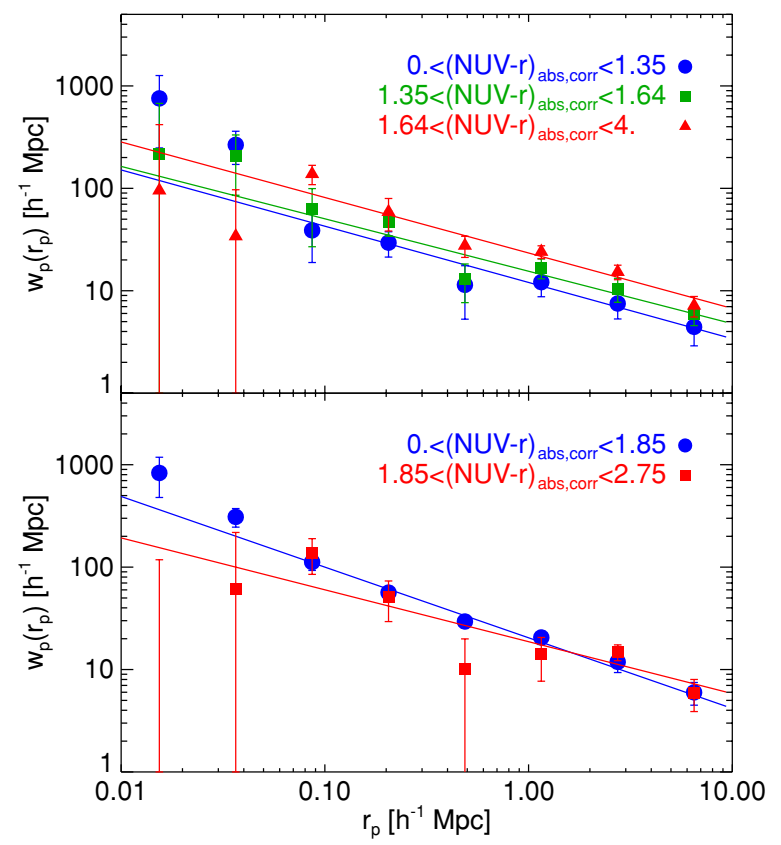

Figure 10. Projected correlation functions of AIS UV-selected samples in bins of $(N U V-r)_{\mathrm{abs}}$ color.

(A color version of this figure is available in the online journal.)

able to probe a large dynamic range in terms of clustering strengths, with values of the correlation length $r_{0}$ increasing from $\sim 1.8 h^{-1} \mathrm{Mpc}$ for the bluest galaxies, to $\sim 7.1 h^{-1} \mathrm{Mpc}$ for the reddest. The UV-selected and MIS/SDSS samples follow the same trends. However, the purely UV-selected galaxies from the AIS survey show a weaker clustering strength at a given color. The correlation length from the AIS sample is lower than that obtained from the MIS/SDSS sample if the same cuts are applied. The mean color for the AIS subsample with $0<(N U V-r)_{\text {abs }}<1.85$ is lower than the corresponding one for the MIS, but the conclusion holds if different cuts are applied and the mean color is similar.

\subsection{Comparison with Previous Studies}

To assess the impact of the NUV selection on our MIS/ SDSS sample, we can directly compare our results with those of Zehavi et al. (2002), who divided their sample between blue and red galaxies using the limit $(u-r)_{\mathrm{abs}}=1.8$. They found for red galaxies $r_{0}=6.78 \pm 0.23 h^{-1} \mathrm{Mpc}, \gamma=1.86 \pm 0.03$ and for blue galaxies $r_{0}=4.02 \pm 0.25 h^{-1} \mathrm{Mpc}, \gamma=1.41 \pm 0.04$. Applying the same cuts to our MIS/SDSS sample, we obtain for red galaxies $r_{0}=5.20 \pm 0.15 h^{-1} \mathrm{Mpc}, \gamma=1.77 \pm 0.02$ and for blue galaxies $r_{0}=3.24 \pm 0.17 h^{-1} \mathrm{Mpc}, \gamma=1.66 \pm 0.07$. We find the same trends as Zehavi et al. (2002) with lower correlation lengths.

We observe a steepening of the slope from $\gamma=1.58 \pm 0.05$ for the bluest galaxies to $\gamma=1.84 \pm 0.03$ for the reddest

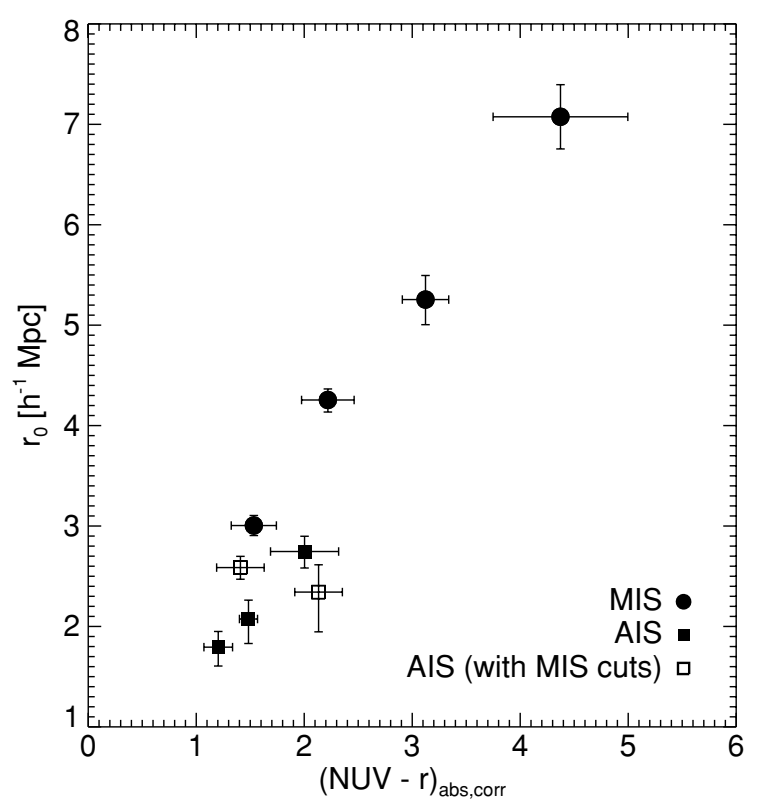

Figure 11. Dependence of the correlation length $r_{0}$ on the $(N U V-r)_{\mathrm{abs}, \mathrm{corr}}$ color. The error bars on color are the standard deviation of the colors in each bin. The filled circles show the MIS/SDSS sample results. The squares show the AIS (UV-selected sample) results: open squares using the same cuts as for the MIS/SDSS sample, and filled squares cuts defined in order to have similar number of galaxies per subsample.

for the results from the MIS/SDSS sample without the dust extinction correction. This increase of the slope with color is similar to what has been already observed by previous studies from optical samples at low redshift. Selecting active and passive galaxies from their spectra, Madgwick et al. (2003) found that they exhibit power laws with respective slopes $\gamma=1.60 \pm 0.04$ and $\gamma=1.95 \pm 0.03$. Budavári et al. (2003) observed similar trends in their angular correlation function measurements as a function of spectral type. Their blue samples have a mean slope $\gamma=1.67 \pm 0.11$ and their red samples have $\gamma=1.99 \pm 0.06$.

The correlation length we obtained from the MIS/SDSS sample for the bluest galaxies in $(N U V-r)_{\mathrm{abs}}$ of $r_{0} \sim$ $3.0 \pm 0.1 h^{-1} \mathrm{Mpc}$ is lower than the values of 3.6-4.5 $h^{-1} \mathrm{Mpc}$ found for blue galaxies in optical samples such as the SDSS (Budavári et al. 2003; Zehavi et al. 2005) or 2dF (Madgwick et al. 2003; Norberg et al. 2002) at similar redshifts. This lower value is due to the combination of our selection which biases against low stellar mass and low SSFR objects, and the larger star formation history dynamic range probed by the $(N U V-r)_{\text {abs }}$ color as well.

The $r_{0}$ value we measured for the bluest bin of our UVselected sample, $r_{0}=1.79 \pm 0.17 h^{-1} \mathrm{Mpc}$, is among the lowest observed in the local universe. It is slightly lower than the value $r_{0}=2.31_{-0.4}^{+0.5} h^{-1} \mathrm{Mpc}$ we obtained from a flux-limited $(N U V<21)$ UV-selected sample (Milliard et al. 2007). 


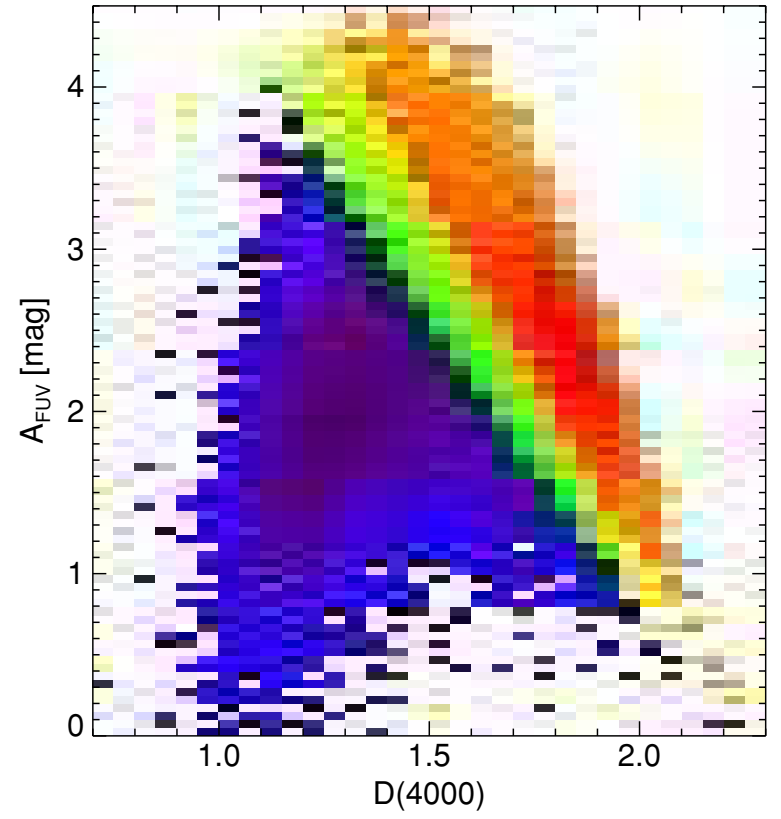

Figure 12. Extinction correction in the FUV band, $A_{\mathrm{FUV}}$, as a function of the $4000 \AA$ break, $D(4000)$ for the MIS/SDSS sample. The extinction correction is obtained from Equation (1). The three color shades code objects on the blue sequence, green valley, and red sequence, from left to right.

(A color version of this figure is available in the online journal.)

These estimates are closer to the values derived for gasrich, Hi-selected galaxies obtained by Meyer et al. (2007) of $r_{0}=2.70 \pm 0.21 h^{-1} \mathrm{Mpc}$ and $\gamma=1.56 \pm 0.1$. This correspondence might indicate that we can associate the bluest galaxies with gas-rich galaxies, which is in agreement with the fact that the gas fraction of blue galaxies is larger (see, e.g., Schiminovich et al. 2007).

Transition galaxies in the green valley indeed exhibit an intermediate clustering pattern, although comparison with other studies might lead to a different conclusion depending on the selections used. For instance, the correlation length we measure is close to that derived by Zehavi et al. (2005) for red galaxies selected by $g-r$ color and $-20<\mathrm{r}_{\text {abs }}<-19$ $\left(r_{0}=5.7 h^{-1} \mathrm{Mpc}\right)$. On the other hand, our $r_{0}$ value for the green valley is also similar to one of the faint samples of blue galaxies studied by Budavári et al. (2003; T3 sample, $r_{0}=5.9 \pm 0.51 h^{-1} \mathrm{Mpc}$ ), who selected galaxies according to their spectral energy distribution. At $z \sim 0.85$ Coil et al. (2008) measured the projected correlation function of green galaxies, selected using the $(U-B)$ rest-frame color. The correlation length of $r_{0}=5.17 \pm 0.42 h^{-1} \mathrm{Mpc}$ that they derived is similar to that we obtained.

\section{DISCUSSION}

\subsection{Intermediate Clustering Strength of Green Valley Galaxies}

The results presented in Section 4 show that green valley galaxies have an intermediate clustering strength compared to bluer or redder galaxies. This holds whether dust extinction is applied or not. As the green valley is a mix of intermediate star formation history galaxies and dusty star-forming ones, interpretation of this result relies on the efficiency of the dust correction procedure to distinguish between these populations. Figure 12 shows the extinction correction in the FUV band, $A_{\text {FUV }}$ as a function of the $4000 \AA$ break, $D(4000)$ for the MIS/SDSS sample. Galaxies within the green valley $(3.75<$
$\left.(N U V-r)_{\mathrm{abs}}<4.5\right)$ lie in the middle (green) region of this plot. This figure shows that the dust correction prescription we used takes into account the evolutionary stage of the galaxy population. For instance, more evolved galaxies with high $D(4000)$ or low SSFR have a lower extinction correction than younger (low $D(4000)$ ) or more active galaxies (high SSFRs), in agreement with recent analysis from Cortese et al. (2008). While still subject to some uncertainties, this procedure should clean up the green valley population from the highly dusty population.

Martin et al. (2007) noted that the fraction of galaxies with AGNs (in their case type 2, or narrow-line AGNs) peaks in the green valley. There is no study devoted to the clustering of type 2 AGNs to which we can directly compare our results. Li et al. (2006b), however, studied the cross-correlation of narrow-line AGNs with galaxies within SDSS. In particular, they showed that their clustering is in agreement with the assumption that a high fraction of them are within central galaxies in dark matter halos, and that they reside mainly within halos with masses $10^{12}-10^{13} M_{\odot}$, values consistent with our results for the green valley (see Section 5.2). Note also that several studies pointed out the "intermediate" nature of galaxies hosting AGNs (Heckman et al. 2004; Kauffmann et al. 2003b). For instance, the average spectra of AGN galaxies host is remarkably similar to those of disk galaxies with Sb Hubble type (Yip et al. 2004a, 2004b).

The intermediate clustering we measured for transition galaxies may be then mainly driven by galaxies hosting AGNs. To test this further, we restricted our MIS/SDSS sample to the overlap with SDSS DR4, in order to discriminate, within the green valley, AGNs and non-AGNs, based on the catalogs from Kauffmann et al. (2003c). Kauffmann et al. (2003c) selected type 2 AGNs from star-forming galaxies using the diagram proposed by Baldwin et al. (1981). We note that the objects classified as "AGNs" by Kauffmann et al. (2003c) include starforming/AGNs composites; the low numbers of this sample do not enable to perform clustering measurement separately on these populations.

We computed the clustering of galaxies within the green valley as defined in Section 4 and using dust correction, but separately for galaxies with or without AGNs. The AGNs represent $56 \%$ of the green valley. The projected correlation functions are presented in Figure 13. The main differences in terms of clustering between these two population, as noted already by $\mathrm{Li}$ et al. (2006b), are at small scales. At scales $0.1<r_{p}<0.5 h^{-1} \mathrm{Mpc}$, the non-AGNs galaxies exhibit marginally more pairs than the AGNs. At large scales, the clustering of AGNs and non-AGNs populations is very similar; the correlation length for the AGNs is $4.7 \pm 0.3 h^{-1} \mathrm{Mpc}$, and the correlation length for the non-AGNs is $5.1 \pm 0.4 h^{-1} \mathrm{Mpc}$. This suggests that the intermediate clustering of the green valley galaxies is genuine and not linked to the predominance of AGNs in this region of the UV-optical color-magnitude diagram.

\subsection{Dark Matter Halo Mass}

We used the analytical models of Mo \& White (2002) to estimate the minimum mass of the dark matter halos that have similar clustering, by matching the bias parameter $b_{8}$, computed at $8 h^{-1} \mathrm{Mpc}$, following, e.g., Magliocchetti et al. (2000). This comparison shows that the mass of the host dark matter halos increases smoothly with $(N U V-r)_{\text {abs. }}$ Blue galaxies have clustering similar to halos with minimum masses around $10^{11} h^{-1} M_{\odot}$, green valley galaxies $10^{12.5} h^{-1} M_{\odot}$, and red galaxies $10^{13} h^{-1} M_{\odot}$. The minimum halo mass for blue galax- 


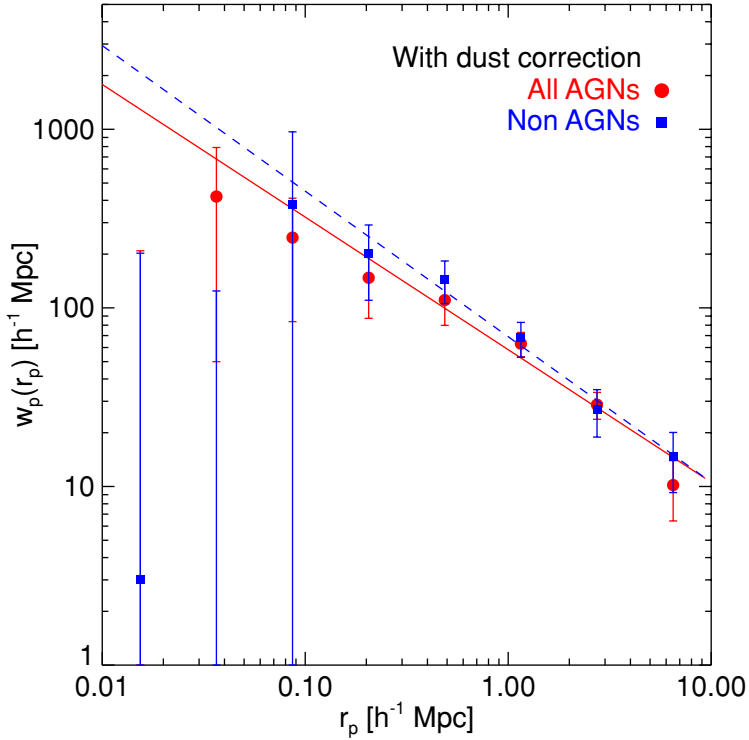

Figure 13. Projected correlation functions for MIS green valley galaxies, assuming dust extinction correction. The sample is here restricted to the overlap with SDSS DR4, and galaxies divided between galaxies with (filled circles) or without AGNs (filled squares).

(A color version of this figure is available in the online journal.)

ies is in agreement with estimates derived from UV-selected galaxies at low redshift (Heinis et al. 2007; Milliard et al. 2007), and is additional evidence that active star formation preferentially occurs in low-density environments at low redshifts (Gómez et al. 2003; Lewis et al. 2002).

The minimum dark halo mass for transition galaxies is similar to those of galaxy groups. This suggests that galaxies within the green valley mainly reside in groups. At $z \sim 0.85$ green valley galaxies have a higher bias, $b=1.65 \pm 0.26$ (Coil et al. 2008); at these redshifts this also corresponds to halos with minimum masses of the order of $10^{12.5} h^{-1} M_{\odot}$. The combination of these results show that green valley galaxies reside in groups of similar masses at $z \sim 0.85$ and $z=0$. We also note that within the green valley the correlation function of star-forming galaxies is marginally larger at small scales than the AGN correlation function (see Figure 13). This could provide some hints about the nature of those objects in the context of the Halo Occupation Distribution (HOD) and the satellite versus central galaxy population. As AGNs are expected to be preferentially central galaxies ( $\mathrm{Li}$ et al. 2006b) this is a hint that transition galaxies could be mainly satellites rather than central galaxies within groups and may preferentially lie in the outskirts of their host DMHs as a recently accreted population, as proposed by Coil et al. (2008). We can speculate on the nature of the mechanisms besides AGN feedback involved in the quenching of star formation in transition galaxies, if they are related to their environment. In this case, given the low relative velocities in galaxy groups, local processes such as tidal mechanisms may induce significant suppression of star formation (e.g., Boselli \& Gavazzi 2006; Bower \& Balogh 2004; Gómez et al. 2003; Lewis et al. 2002).

Finally, as expected, the reddest galaxies have a clustering strength similar to large groups or clusters.

These results show that there is a correlation between star formation history and dark matter halo mass, as the galaxies having formed stars more recently inhabit less massive halos. These findings are in agreement with the "downsizing" scenario (Cowie et al. 1996), which states that more massive galaxies

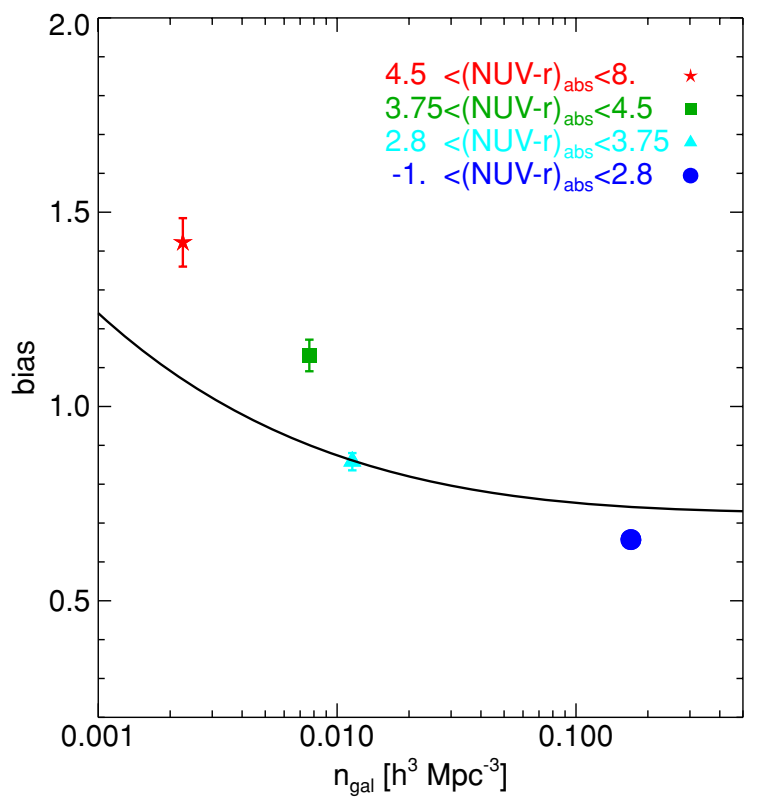

Figure 14. Bias as a function of galaxy number density for $(N U V-r)_{\mathrm{abs}}$ cut samples (same color legend as Figure 8). Here we show only results without the dust extinction correction. The solid line shows the relation expected for dark matter halos, using Mo \& White (2002) models.

(A color version of this figure is available in the online journal.)

formed their stars at higher redshifts (see, e.g., Bundy et al. 2006; De Lucia et al. 2006).

\subsection{Halo Occupation Limits}

Our approach in the previous section to estimate the dark matter halo mass from the clustering with Mo \& White (2002) models has the caveat that it assumes only one galaxy per halo. This assumption is quite unlikely according to recent numerical and observational studies (e.g., Kravtsov et al. 2004; Ouchi et al. 2005). In the framework of the HOD (e.g., Cooray \& Sheth 2002), the number of galaxies in a given halo depends on its mass. We can, however, use Mo \& White (2002) models to get a first estimate of the dependence of the halo occupation with star formation history. In Figure 14, we compare the bias and the number density of the samples with those of the dark matter halos. We computed the number densities of the various samples using the luminosity functions derived by Wyder et al. (2007) in $(N U V-r)_{\mathrm{abs}}$ bins from a similar MIS sample. These luminosity functions are not available for dust extinction corrected samples, so we only show on Figure 14 our MIS results obtained without any dust correction. As the clustering results are in very good agreement with or without applying the dust correction, and as we want to get only some qualitative limits on the halo occupation numbers, we expect our conclusions to be robust.

The relation expected for dark matter halos from the Mo \& White (2002) models is shown by the solid line. On this plot, the dark matter halo mass increases on this line from right to left. As this model assumes that each halo hosts one galaxy, measurements on the solid line indicate an average halo occupation number of 1 , measurements above an average halo occupation greater than 1 , and measurements below an average occupation lower than 1 . Our measurements recover the trend expected for dark matter halos, as galaxy populations with lower number densities are more clustered. However, galaxies do not lie on the dark matter halos bias-density relation, which is due to the fact that the halo occupation varies among 


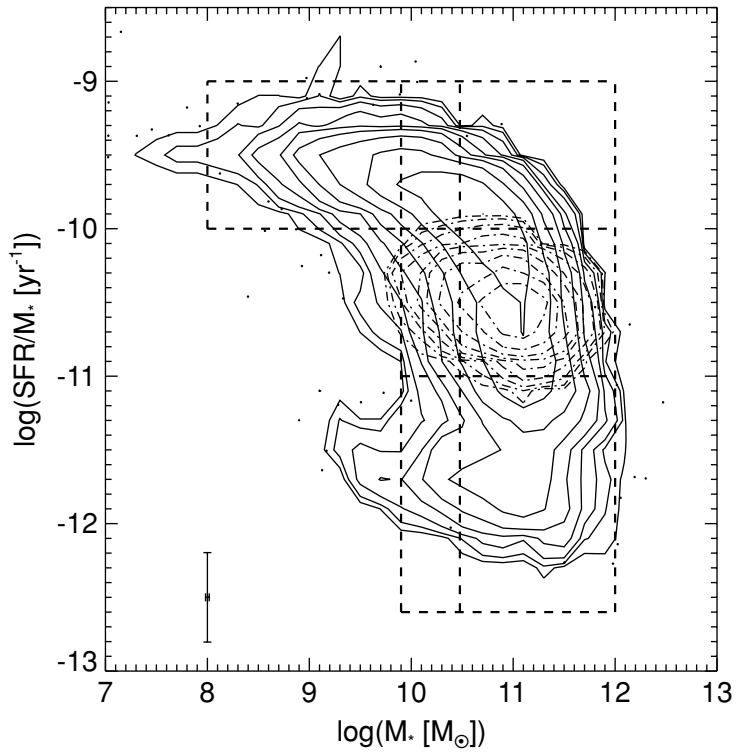

Figure 15. SSFR as a function of stellar mass. The dot-dashed contours show the distribution of the green valley defined with $2.75<(N U V-r)_{\mathrm{abs}, \mathrm{corr}}<3.5$. The dashed lines represent the cuts we consider for clustering measurements. The error bars show the dispersion caused by the dust extinction correction.

populations. Our results show that the average occupation number increases with $(N U V-r)_{\text {abs }}$ color. Bluest galaxies in the local universe have a very low occupation number in halos that have similar clustering strength. Our second blue sample contains mainly galaxies with an average occupation number close to 1. Transition and red galaxies have an occupation number around 5. Note that the occupation number for the red galaxies is potentially an underestimation given that our selection biases against low mass and low SSFRs galaxies (see Section 4.2)

These results are in qualitative agreements with numeric (Berlind et al. 2003; Zheng et al. 2005), as well as observational studies (Hogg et al. 2003; Magliocchetti \& Porciani 2003; Zehavi et al. 2005).

\subsection{Specific Star Formation Rate versus Stellar Mass}

The results we present above provide further evidence that the clustering of galaxies strongly depends on star formation history. However, galaxy clustering also depends on stellar mass (e.g., Li et al. 2006a; Meneux et al. 2008); we investigate which of these two parameters shows the strongest correlation with environment. To do so, we consider an alternative to the UVoptical color-magnitude diagram, by plotting the specific star formation rate $S F R / M_{*}$ as a function of the stellar mass $M_{*}$ in Figure 15.

We computed the SFRs from the NUV luminosities corrected for dust extinction (see Section 2.3) using the Kennicutt (1998) relation scaled to a Kroupa (2001) initial mass function:

$$
\operatorname{SFR}\left(M_{\odot} \mathrm{yr}^{-1}\right)=10^{-28.02} L_{v}\left(\operatorname{erg~s}^{-1} \mathrm{~Hz}^{-1}\right) .
$$

We estimate the stellar masses using the equation derived by Yang et al. (2007) from the relation between stellar mass-to-light ratio and color of Bell et al. (2003):

$$
\begin{aligned}
\log \left[\frac{M_{*}}{M_{\odot}}\right]= & -0.306+1.097\left[\left(g_{\mathrm{abs}}-r_{\mathrm{abs}}\right)_{\mathrm{corr}}\right] \\
& -0.1-0.4\left[r_{\mathrm{abs}, \mathrm{corr}}-4.64\right]
\end{aligned}
$$

which implicitly assumes a Kroupa (2001) initial mass function.

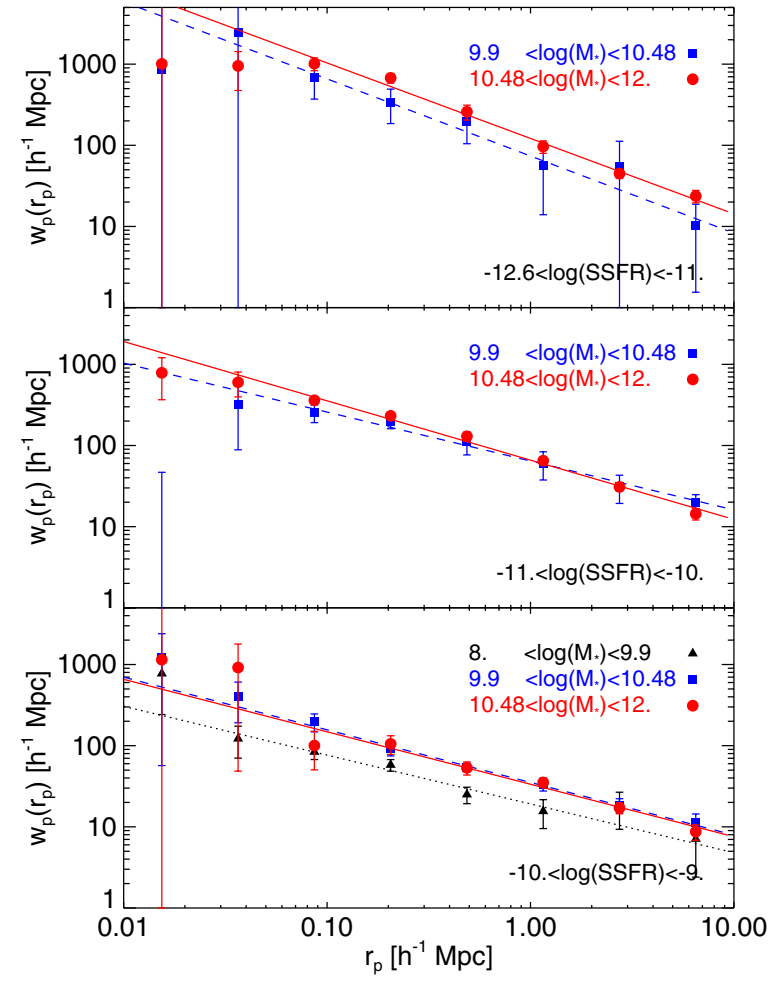

Figure 16. Projected correlation functions of the MIS/SDSS samples cut by stellar mass and SSFR. The top panel shows measurements obtained for the $-12.6<\log (S S F R)<-11$ bin, the middle panel for the $-11<\log (S S F R)<$ $-10 \mathrm{bin}$, and the bottom panel for the $-10<\log (S S F R)<-9 \mathrm{bin}$. On each panel, filled squares show results from $9.9<\log \left(M_{*}\right)<10.48$, filled circles from $10.48<\log \left(M_{*}\right)<12$, and filled triangles $8<\log \left(M_{*}\right)<9.9$ (only for $-10<\log (S S F R)<-9)$.

(A color version of this figure is available in the online journal.)

The cuts we adopted are shown as dashed lines in Figure 15. Within the stellar mass range $10^{9.9}<M_{*}<10^{12} M_{\odot}$, we choose an additional mass cut at $M_{*}=3 \times 10^{10} M_{\odot}$. Several studies indeed showed that in the local universe this value divides galaxies between active or passive in terms of star formation (Kauffmann et al. 2003a). Moreover, according to numerical studies (Kereš et al. 2005, 2009), the way galaxies accrete gas depends on stellar mass. Galaxies below $3 \times 10^{10} M_{\odot}$ are dominated by a "cold mode" of gas accretion, while galaxies above this mass are dominated by a "hot mode" of gas accretion. The geometry of these two modes is also different, the cold mode being mostly filamentary, while the hot mode quasi-spherical, hence implying a link with environment. We then further divide our subsample in mass by this value $M_{*}=3 \times 10^{10} M_{\odot}$, and also use two cuts in SSFR at $\log \left(S F R / M_{*}\left[\mathrm{yr}^{-1}\right]\right)=10^{-11}$ and $\log \left(S F R / M_{*}\left[\mathrm{yr}^{-1}\right]\right)=10^{-10}$.

The correlation functions are shown Figure 16 and the clustering parameters are listed in Table 4. Each panel of Figure 16 shows a given cut in SSFR, and the relevant cuts in stellar mass. While there are some differences in the small-scale clustering ( $r_{p} \lesssim 0.5 h^{-1} \mathrm{Mpc}$ ), at larger scales the correlation functions for the galaxies with $10^{9.9}<M_{*}<10^{12} M_{\odot}$ are remarkably similar for a given SSFR. The main difference we observe in clustering strength is for the less massive sample $\left(10^{8 .}<M_{*}<10^{9.9} M_{\odot}\right)$ within the highest SSFR bin. There is also a difference for the lowest SSFR bin, but in this bin the low mass sample with $10^{9.9}<M_{*}<10^{10.48} M_{\odot}$ has very low statistics so its correlation function must be interpreted with caution. 
Table 4

MIS/SDSS Sample $M_{*}-S S F R$ Clustering Results ${ }^{\mathrm{a}}$

\begin{tabular}{cccccccc}
\hline \hline \multicolumn{1}{c}{ Sample $^{\mathrm{b}}$} & & $N_{\text {gal }}$ & $\langle z\rangle$ & $\left\langle M_{*}\right\rangle\left(M_{\odot}\right)$ & $r_{0}\left(h^{-1} \mathrm{Mpc}\right)$ & $\gamma$ & $b_{8}$ \\
\hline \hline$-10 .<\log \left(\right.$ SFR $\left./ M_{*}\right)<-9$. & $10^{8 .}<M_{*}<10^{9.9}$ & 2103 & 0.04 & $10^{9.56}$ & $2.46 \pm 0.21$ & $1.6 \pm 0.1$ & $0.56 \pm 0.04$ \\
& $10^{9.9}<M_{*}<10^{10.48}$ & 3347 & 0.07 & $10^{10.24}$ & $3.58 \pm 0.17$ & $1.65 \pm 0.07$ & $0.76 \pm 0.03$ \\
& $10^{10.48}<M_{*}<10^{12 .}$ & 3375 & 0.12 & $10^{10.82}$ & $3.48 \pm 0.16$ & $1.65 \pm 0.06$ & $0.76 \pm 0.03$ \\
$-11 .<\log \left(\right.$ SFR $\left./ M_{*}\right)<-10$. & $10^{9.9}<M_{*}<10^{10.48}$ & 1142 & 0.06 & $10^{10.32}$ & $5.23 \pm 0.4$ & $1.60 \pm 0.06$ & $1.03 \pm 0.06$ \\
& $10^{10.48}<M_{*}<10^{12 .}$ & 8461 & 0.11 & $10^{11.07}$ & $5.10 \pm 0.17$ & $1.73 \pm 0.03$ & $1.06 \pm 0.03$ \\
$-12.6<\log \left(\right.$ SFR $\left./ M_{*}\right)<-11$. & $10^{9.9}<M_{*}<10^{10.48}$ & 304 & 0.05 & $10^{10.30}$ & $4.95 \pm 0.9$ & $1.95_{-0.05}^{+0.15}$ & $1.04 \pm 0.18$ \\
& $10^{10.48}<M_{*}<10^{12 .}$ & 2925 & 0.10 & $10^{11.23}$ & $6.47 \pm 0.29$ & $1.93 \pm 0.04$ & $1.38 \pm 0.06$ \\
\hline
\end{tabular}

Notes.

${ }^{a}$ With dust extinction correction.

b The specific star formation rate, $S F R / M_{*}$ is in units of $\mathrm{yr}^{-1}$.

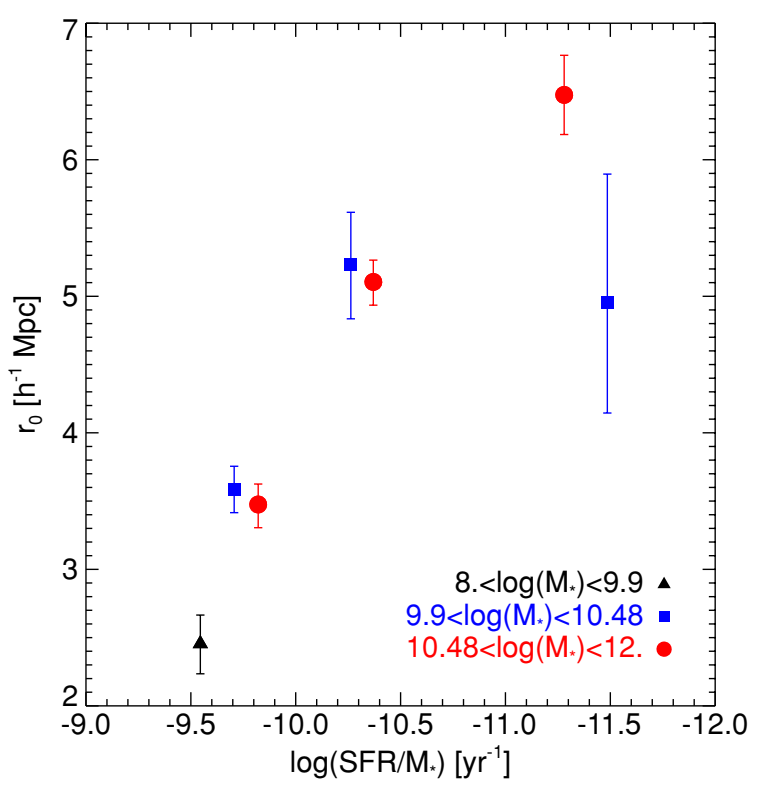

Figure 17. Correlation length as a function of SSFR and stellar mass. The filled triangle shows the result from $8 .<\log \left(M_{*}\right)<9.9$, filled squares show results from $9.9<\log \left(M_{*}\right)<10.48$, and filled circles from $10.48<\log \left(M_{*}\right)<12$.

(A color version of this figure is available in the online journal.)

We show in Figure 17 the correlation lengths derived from these measurements as a function of SSFR and stellar mass. The global trend is that $r_{0}$ decreases with SSFR. For $-11<\log$ $\left(S F R / M_{*}\right)<-9$ and $9.9<\log \left(M_{*}\right)<12$, the correlation length does not depend on stellar mass in a given SSFR range. This does not seem to be true in our extreme stellar mass bins. However, the statistics of our $-12.6<\log \left(S F R / M_{*}\right)<$ $-11, \quad 9.9<\log \left(M_{*}\right)<10.48$ sample are small; and because of our selection, we cannot compare to lower SSFRs at 8 . < $\log \left(M_{*}\right)<9.9$. Nevertheless, these results suggest that the SSFR shows a stronger correlation with environment (as traced by the large-scale clustering) than the stellar mass. Interestingly, this is true below and above the transition mass of $3 \times 10^{10} M_{\odot}$. Within the ranges $-11<\log \left(S F R / M_{*}\right)<-9$ and $9.9<$ $\log \left(M_{*}\right)<12$, our results show that there is a smooth transition of the large-scale clustering strength, and hence in dark matter host halo masses, as a function of star formation history, independently of stellar mass. This result is consistent with our measurements based on color selected samples (because of the correlation between specific SSFR and $\left.(N U V-r)_{\mathrm{abs}}\right)$, but provides a direct interpretation of the dependence of clustering on galaxies physical parameters (stellar mass and SSFR).
In the local universe, high SSFR galaxies are anti-biased $\left(b_{8}<1\right)$, and have clustering similar to dark matter halos of minimum masses $\sim 10^{11} M_{\odot}$. Galaxies with intermediate SSFRs and within the green valley are unbiased $\left(b_{8} \sim 1\right)$, and are hosted by dark matter halos of minimum masses $\sim 10^{12} M_{\odot}$. Lower SSFR galaxies are biased $\left(b_{8}>1\right)$; their clustering strength is similar to dark matter halos of minimum masses $\sim 10^{13} M_{\odot}$.

These results are in agreements with those of Blanton et al. (2005) who noticed that color $(g-r$ in their case, which is also a tracer of star formation history) is the galaxy property most predictive of environment. Kauffmann et al. (2004) showed that the galaxy property most sensitive to environment is star formation history (and in particular the SSFR), and although the amplitude of the dependence varies with mass, it is observed at all masses (for $10^{9.5}<M_{*}<10^{11} M_{\odot}$ ).

\section{CONCLUSIONS}

We used the unique combination of GALEX UV photometry and SDSS spectroscopy to perform spatial clustering studies from UV and UV/optical-selected samples. We take advantage of the remarkable correlation between the $(N U V-r)_{\text {abs }}$ color with star formation history to directly investigate how it relates to large-scale clustering.

The results from the UV-selected samples, in addition to those from Heinis et al. (2007) and Milliard et al. (2007), confirm the low clustering of UV-selected galaxies in the local universe. This is further evidence that active star formation takes preferentially place in low density environments at low redshifts (Gómez et al. 2003; Lewis et al. 2002). Within the blue sequence, redder UVselected galaxies are more clustered, but the clustering strength of UV-selected galaxies is, however, lower than for opticallyselected galaxies at a given $(N U V-r)_{\text {abs }}$ color.

The results from our MIS/SDSS sample recover the expected trends from previous studies that redder galaxies are more clustered. Thanks to the greater sensitivity of the $(N U V-r)_{\mathrm{abs}}$ color to star formation history than optical colors, our results span a wide range in clustering strength. Our results show that galaxies that experienced star formation more recently inhabit less massive halos, which is consistent with the "downsizing" scenario (e.g., Bundy et al. 2006; Cowie et al. 1996; Neistein et al. 2006). Also, their average halo occupation is lower, in such a way that the bluest galaxies are hosted by only a small fraction of the dark matter halos that have the same clustering. These findings show that, in contrast to what is observed from Lyman break galaxies at high redshift (see, e.g., Giavalisco \& Dickinson 2001; Lee et al. 2006), star formation is not primarily 
driven by gravitation in the local universe. The clustering of the green valley galaxies suggests that they are mainly satellites within galaxy groups. Comparison with previous work of Coil et al. (2008) indicates that these transition galaxies reside in similar environments at $z \sim 1$ and $z=0$.

Our results show that there are smooth trends between star formation history and environment. It is interesting to note that based on the results from optical selections, one might not expect to observe such smooth transitions. Previous studies showed that there is a bimodality in a number of galaxy properties: color ( $u-r$ for instance; see Baldry et al. 2004; Strateva et al. 2001), stellar mass (Kauffmann et al. 2003b), or clustering (Budavári et al. 2003). However, Wyder et al. (2007) already noticed that the $(N U V-r)_{\text {abs }}$ color distribution in the local universe is not as bimodal as the $u-r$ color (see also our Figure 6), due to the greater sensitivity of the UV spectral range to star formation activity, and reddening by interstellar dust. Our results suggest that in the local universe, rather than a bimodality, there is a smooth transition in global environments (as probed by the clustering) for galaxies with different star formation histories (as probed by the $(N U V-r)_{\text {abs }}$ color). These results hold whether dust extinction is applied or not, and are insensitive to the predominance of AGNs among transition galaxies.

Finally, our results show that the large-scale clustering trends we observe are mainly driven by star formation history, and not stellar mass. This is indication that at a fixed star formation history, there is no strong variation of the host dark matter halo mass. Our results suggest that the environmental dependence is first driven by the star formation history and that the stellar mass only acts as a secondary parameter.

We thank Ching-Wa Yip for useful discussions. GALEX (Galaxy Evolution Explorer) is a NASA Small Explorer, launched in 2003 April. We gratefully acknowledge NASAs support for construction, operation, and science analysis for the GALEX mission, developed in cooperation with the Centre National $\mathrm{d}(\mathrm{tm})$ Etudes Spatiales of France and the Korean Ministry of Science and Technology.

Facilities: GALEX, Sloan

\section{REFERENCES}

Adelman-McCarthy, J. K., et al. 2008, ApJS, 175, 29

Arnouts, S., et al. 2007, A\&A, 476, 137

Baldry, I. K., Glazebrook, K., Brinkmann, J., Ivezić, Ž., Lupton, R. H., Nichol, R. C., \& Szalay, A. S. 2004, ApJ, 600, 681

Baldwin, J. A., Phillips, M. M., \& Terlevich, R. 1981, PASP, 93, 5

Balogh, M. L., Baldry, I. K., Nichol, R., Miller, C., Bower, R., \& Glazebrook, K. 2004, ApJ, 615, L101

Bell, E. F., McIntosh, D. H., Katz, N., \& Weinberg, M. D. 2003, ApJS, 149, 289

Bell, E. F., et al. 2004, ApJ, 608, 752

Berlind, A. A., et al. 2003, ApJ, 593, 1

Blanton, M. R., Eisenstein, D., Hogg, D. W., Schlegel, D. J., \& Brinkmann, J. 2005, ApJ, 629, 143

Boselli, A., \& Gavazzi, G. 2006, PASP, 118, 517

Bower, R. G., \& Balogh, M. L. 2004, Clusters of Galaxies: Probes of Cosmological Structure and Galaxy Evolution, ed. J. S. Mulchaey, A. Dressler, \& A. Oemler (Cambridge: Cambridge Univ. Press), 325

Bruzual, G., \& Charlot, S. 2003, MNRAS, 344, 1000

Budavári, T., et al. 2003, ApJ, 595, 59

Budavári, T., et al. 2008, in ASP Conf. Ser. 382, The National Virtual Observatory Book, ed. M. J. Graham, M. J. Fitzpatrick, \& T. A. McGlynn (San Francisco, CA: ASP), 75

Budavári, T., et al. 2009, ApJ, 694, 1281

Bundy, K., et al. 2006, ApJ, 651, 120

Calzetti, D., Armus, L., Bohlin, R. C., Kinney, A. L., Koornneef, J., \& StorchiBergmann, T. 2000, ApJ, 533, 682

Cassata, P., et al. 2008, A\&A, 483, L39
Chung, A., van Gorkom, J. H., Kenney, J. D. P., \& Vollmer, B. 2007, ApJ, 659 L115

Cirasuolo, M., et al. 2007, MNRAS, 380, 585

Coil, A. L., et al. 2008, ApJ, 672, 153

Cooray, A., \& Sheth, R. 2002, Phys. Rep., 372, 1

Cortese, L., Boselli, A., Franzetti, P., Decarli, R., Gavazzi, G., Boissier, S., \& Buat, V. 2008, MNRAS, 386, 1157

Cowie, L. L., Songaila, A., Hu, E. M., \& Cohen, J. G. 1996, AJ, 112, 839

Croton, D. J., et al. 2006, MNRAS, 365, 11

Cucciati, O., et al. 2006, A\&A, 458, 39

Davis, M., \& Peebles, P. J. E. 1983, ApJ, 267, 465

Dekel, A., \& Silk, J. 1986, ApJ, 303, 39

De Lucia, G., Springel, V., White, S. D. M., Croton, D., \& Kauffmann, G. 2006, MNRAS, 366, 499

Faber, S. M., et al. 2007, ApJ, 665, 265

Fisher, K. B., Davis, M., Strauss, M. A., Yahil, A., \& Huchra, J. 1994, MNRAS 266, 50

Franzetti, P., et al. 2007, A\&A, 465, 711

Giavalisco, M., \& Dickinson, M. 2001, ApJ, 550, 177

Gómez, P. L., et al. 2003, ApJ, 584, 210

Gott, J. R. I., Jurić, M., Schlegel, D., Hoyle, F., Vogeley, M., Tegmark, M., Bahcall, N., \& Brinkmann, J. 2005, ApJ, 624, 463

Gunn, J. E., \& Gott, J. R. I. 1972, ApJ, 176, 1

Hawkins, E., et al. 2003, MNRAS, 346, 78

Heckman, T. M., Kauffmann, G., Brinchmann, J., Charlot, S., Tremonti, C., \& White, S. D. M. 2004, ApJ, 613, 109

Heinis, S., et al. 2007, ApJS, 173, 503

Hogg, D. W., et al. 2003, ApJ, 585, L5

Hopkins, A. M., \& Beacom, J. F. 2006, ApJ, 651, 142

Hopkins, P. F., Bundy, K., Hernquist, L., \& Ellis, R. S. 2007, ApJ, 659 976

Johnson, B. D., et al. 2006, ApJ, 644, L109

Juneau, S., et al. 2005, ApJ, 619, L135

Kaiser, N. 1987, MNRAS, 227, 1

Kauffmann, G., et al. 2003a, MNRAS, 341, 33

Kauffmann, G., et al. 2003b, MNRAS, 341, 54

Kauffmann, G., et al. 2003c, MNRAS, 346, 1055

Kauffmann, G., White, S. D. M., Heckman, T. M., Ménard, B., Brinchmann, J., Charlot, S., Tremonti, C., \& Brinkmann, J. 2004, MNRAS, 353 713

Kennicutt, R. C., Jr. 1998, ARA\&A, 36, 189

Kereš, D., Katz, N., Fardal, M., Dave, R., \& Weinberg, D. H. 2009, MNRAS, 395,160

Kereš, D., Katz, N., Weinberg, D. H., \& Davé, R. 2005, MNRAS, 363, 2

Khochfar, S., \& Ostriker, J. P. 2008, ApJ, 680, 54

Kravtsov, A. V., Berlind, A. A., Wechsler, R. H., Klypin, A. A., Gottlöber, S. Allgood, B., \& Primack, J. R. 2004, ApJ, 609, 35

Kroupa, P. 2001, MNRAS, 322, 231

Landy, S. D., \& Szalay, A. S. 1993, ApJ, 412, 64

Larson, R. B., Tinsley, B. M., \& Caldwell, C. N. 1980, ApJ, 237, 692

Lee, K.-S., Giavalisco, M., Gnedin, O. Y., Somerville, R. S., Fergguson, H. C., Dickinson, M., \& Ouchi, M. 2006, ApJ, 642, 63

Lewis, I., et al. 2002, MNRAS, 334, 673

Li, C., Kauffmann, G., Jing, Y. P., White, S. D. M., Börner, G., \& Cheng, F. Z 2006a, MNRAS, 368, 21

Li, C., Kauffmann, G., Wang, L., White, S. D. M., Heckman, T. M., \& Jing, Y. P. 2006b, MNRAS, 373, 457

Madgwick, D. S., et al. 2003, MNRAS, 344, 847

Magliocchetti, M., Bagla, J. S., Maddox, S. J., \& Lahav, O. 2000, MNRAS, 314,546

Magliocchetti, M., \& Porciani, C. 2003, MNRAS, 346, 186

Martin, D. C., et al. 2005, ApJ, 619, L1

Martin, D. C., et al. 2007, ApJS, 173, 342

Meneux, B., et al. 2008, A\&A, 478, 299

Meyer, M. J., Zwaan, M. A., Webster, R. L., Brown, M. J. I., \& Staveley-Smith L. 2007, ApJ, 654, 702

Milliard, B., et al. 2007, ApJS, 173, 494

Mo, H. J., \& White, S. D. M. 2002, MNRAS, 336, 112

Moore, B., Lake, G., Quinn, T., \& Stadel, J. 1999, MNRAS, 304, 465

Morrissey, P., et al. 2005, ApJ, 619, L7

Morrissey, P., et al. 2007, ApJS, 173, 682

Neistein, E., van den Bosch, F. C., \& Dekel, A. 2006, MNRAS, 372, 933

Norberg, P., et al. 2002, MNRAS, 332, 827

Ouchi, M., et al. 2005, ApJ, 635, L117

Salim, S., et al. 2005, ApJ, 619, L39

Salim, S., et al. 2007, ApJS, 173, 267

Schawinski, K., et al. 2007, ApJS, 173, 512 
Schiminovich, D., et al. 2005, ApJ, 619, L47

Schiminovich, D., et al. 2007, ApJS, 173, 315

Schlegel, D. J., Finkbeiner, D. P., \& Davis, M. 1998, ApJ, 500, 525

Strateva, I., et al. 2001, AJ, 122, 1861

Strauss, M. A., et al. 2002, AJ, 124, 1810

Tonnesen, S., Bryan, G. L., \& van Gorkom, J. H. 2007, ApJ, 671, 1434

Wyder, T. K., et al. 2007, ApJS, 173, 293
Yang, X., Mo, H. J., van den Bosch, F. C., Pasquali, A., Li, C., \& Barden, M. 2007, ApJ, 671, 153

Yip, C. W., et al. 2004a, AJ, 128, 585

Yip, C. W., et al. 2004b, AJ, 128, 2603

Zehavi, I., et al. 2002, ApJ, 571, 172

Zehavi, I., et al. 2005, ApJ, 630, 1

Zheng, Z., et al. 2005, ApJ, 633, 791 